\title{
Thickenings and Conformal Gravity
}

\author{
Claude LeBrun $\star$ \\ Department of Mathematics, SUNY, Stony Brook, NY 11794, USA \\ Received June 6, 1989
}

\begin{abstract}
A twistor correspondence is given for complex conformal space-times with vanishing Bach and Eastwood-Dighton tensors; when the Weyl curvature is algebraically general, these equations are precisely the conformal version of Einstein's vacuum equations with cosmological constant. This gives a fully curved version of the linearized correspondence of Baston and Mason [B-M].
\end{abstract}

\section{Introduction}

In this paper we provide a twistor correspondence for conformal gravity, meaning roughly a reformulation of the conformally invariant aspects of Einstein's vacuum equations in terms of deformations of complex analytic spaces. This correspondence was conjectured by Baston and Mason [B-M] on the basis of some insightful (albeit heuristic) arguments concerning the linearized theory, and the chief new idea that will be explored here, the rôle of Poisson structures (cf. [W]) in the relevant extension problem, arose directly from the efforts of the present author to give the calculations of Baston and Mason precise meaning.

We work throughout in the context of conformal classes of complex-Riemannian 4-manifolds. Recall that a complex-Riemannian manifold is a complex manifold equipped with a non-degenerate holomorphic symmetric 2-tensor, so that each tangent space is endowed with a complex quadratic form; two such complexRiemannian metrics are called conformally equivalent if one is obtained from the other by multiplication by a non-zero holomorphic function. Such structures arise naturally from the analytic continuation of real-analytic pseudo-Riemannian metrics and their conformal classes into the complex domain, and one may return to the realm of pseudo-Riemannian geometry by restricting to the fixed-point set of an anti-holomorphic involution ("complex conjugation") respecting the structure. While it is possible to reformulate some of our results without this foray into the complex domain, we will avoid so doing here for the sake of brevity.

* Research supported in part by NSF grant DMS-8704401 
Let us now recall the basic ambitwistor correspondence ([L2, L3]) which provides the foundation for the present edifice. Associated to any complex-Riemannian conformal class is the set of complex null geodesics, which are those maximal complex one-dimensional submanifolds whose tangent spaces are null with respect to the conformal structure and auto-parallel with respect to the Levi-Civita connection of some (and hence every) metric in the conformal class. Under mild convexity hypotheses, the set of these null geodesics forms a complex manifold, under which circumstances the conformal manifold is said to be civilized. A civilized complex-Riemannian 4-manifold is often referred to as a complex space-time, and its associated complex 5-manifold of null geodesics is usually called it ambitwistor space.

The whole point of considering the ambitwistor space is that its complex structure completely encodes the conformal structure of the complex space-time. Indeed, if $\mathscr{N}$ is an ambitwistor space, the corresponding space-time $\mathscr{M}$ is precisely the space of complex submanifolds on $\mathscr{N}$ which are biholomorphic to the 2-quadric $Q=\mathbb{P}_{1} \times \mathbb{P}_{1}$ and have normal bundle isomorphic to $N=J^{1} \mathcal{O}(1,1)$, where $\mathcal{O}(1,1)$ denotes the divisor line bundle of the diagonal $\mathbb{P}_{1} \subset \mathbb{P}_{1} \times \mathbb{P}_{1}$. For each $x \in \mathscr{M}$, one obtains such a 2-quadric $Q_{x}$, called the sky of $x$, by considering the set of null geodesics through $x$; conversely, every quadric with the right normal bundle type is of this form. Since any null geodesic in $\mathscr{M}$ corresponds precisely to the set of skies passing through some point of $\mathscr{N}$, our reconstructed version of $\mathscr{M}$ comes equipped with its complex conformal structure.

Given a complex 5-manifold, one might now ask if it is the ambitwistor space for some complex space-time $\mathscr{M}$. In light of the above discussion, a necessary condition is certainly that through each point here must pass at least one quadric $Q=\mathbb{P}_{1} \times \mathbb{P}_{1}$ with normal bundle $N=J^{1} \mathcal{O}(1,1)$. This, however, does not suffice, for an ambitwistor space has one other important property - it admits a (unique!) complex contact structure, meaning a holomorphic distribution of 4-planes $\mathrm{D} \subset T \mathscr{N}$ such that the Frobenius integrability obstruction

$$
\begin{aligned}
A: D \times D & \rightarrow T \mathcal{N} / D \\
(v, w) & \mapsto[v, w]+D
\end{aligned}
$$

is non-degenerate. Equivalently, a contact structure on our complex 5-manifold $\mathscr{N}$ is a line-bundle-valued 1 -form $\theta \in \Gamma \Omega^{1}(L)$ such that $\theta \wedge d \theta \wedge d \theta \in \Gamma \Omega^{5}\left(L^{\otimes 3}\right)$ is non-vanishing; $\theta: T \mathscr{N} \rightarrow L$ is just the canonical projection $T \mathcal{N} \rightarrow(T \mathcal{N} / D)=: L$, and $D=\operatorname{ker} \theta$. It is not difficult to check that such a contact form must vanish when restricted to the tangent bundle of a quadric $Q=\mathbb{P}_{1} \times \mathbb{P}_{1}$ with normal bundle $N=J^{1} \mathcal{O}(1,1)$, and thus the contact distribution $D \subset T \mathscr{N}$ is characterized by the fact that it contains the tangent spaces of all the skies. (If two nearby skies meet, their tangent spaces jointly span a 4-dimensional space at the point of intersection.) The fact that an ambitwistor space does indeed admit a complex contact structure follows from the fact that the null geodesics of $\mathscr{M}$ are precisely given by the Hamiltonian trajectories of the manifold of null covectors. For details cf. [L2].

The ambitwistor space of complexified Minkowski space $\left(\mathbb{C}^{4}, \sum_{j=1}^{4}\left(d z^{j}\right)^{\otimes 2}\right)$ is just the total space of the bundle $N \rightarrow Q$. This has a beautiful compactification as 
the hypersurface $\mathbf{A} \subset \mathbb{P}_{3} \times \mathbb{P}_{3}$ given by

$$
\left.\mathbf{A}=\left\{([Z],[W]) \in \mathbb{P}(T) \times \mathbb{P}\left(T^{*}\right) \mid Z\right\lrcorner W=0\right\},
$$

where $T$ is a complex 4-dimensional vector space and where $\lrcorner$ denotes contraction; correspondingly, complexified Minkowski space compactifies as the 4-quadric $M \cong G_{2}(T)$. The projective 3 -spaces $\mathbb{P}(T)$ and $\mathbb{P}\left(T^{*}\right)$ have an autonomous geometrical meaning, for their disjoint union parameterizes the set of totally null (i.e. isotropic) 2-surfaces in $M$, and the imbedding $\mathbf{A} \subset \mathbb{P}(T) \times \mathbb{P}\left(T^{*}\right)$ reflects the fact that a null geodesic in $M$ is contained in precisely two such 2-surfaces, one of each type. $\mathbb{P}(T)$ and $\mathbb{P}\left(T^{*}\right)$ are respectively known $[\mathbf{P}-\mathbf{R}]$ as the twistor and dual twistor spaces of $M$.

The fact that $\mathbf{A}$ arises so naturally as a hypersurface in a bigger manifold encourages one to consider the sequence of complex analytic spaces

$$
\mathbf{A}^{(m)}=\left(\mathbf{A}, \mathcal{O}_{\mathbb{P}_{3} \times \mathbb{P}_{3}} / \mathscr{T}^{m+1}\right),
$$

where $\mathscr{T}$ is the ideal of holomorphic functions on $\mathbb{P}(T) \times \mathbb{P}\left(T^{*}\right)$ which vanish on A; these analytic spaces are the so-called infinitesimal neighborhoods or thickenings of $\mathbf{A} \subset \mathbb{P}(T) \times \mathbb{P}\left(T^{*}\right)$, and may be thought of as the jets of our imbedding of $\mathbf{A}$ into a larger complex manifold. Their relevance to the PDE's of mathematical physics was first recognized by Isenberg et al. [I-Y-G] and Witten [Wi1], who showed that Yang-Mills fields on complexified Minkowski space correspond to holomorphic vector bundles (i.e. locally free sheaves) on $\mathbf{A}^{(3)}$. The fact that these equations make good sense in curved space-time then seemed to indicate that, at least for small $m$, one might expect the existence of analogs $\mathscr{N}^{(m)}$ of the infinitesimal neighborhoods for any ambitwistor space $\mathcal{N}$, and early on a curved version of $\mathbf{A}^{(1)}$ was, in fact, found [L1]. Later, under the strong additional hypothesis that the space-time $M$ is assumed to be half-conformally flat (i.e. has self-dual or anti-self-dual Weyl curvature), it was shown [L4] that analogs $\mathscr{N}^{(m)}$ of the $\mathbf{A}^{(m)}$ actually exist for all $m$; this was done by exploiting the fact [P] that in these cases an analog exists either for $\mathbb{P}(T)$ or for $\mathbb{P}\left(T^{*}\right)$, depending on whether the Weyl curvature is self-dual or anti-self-dual. For algebraically general conformal curvature, however, analogy with Yang-Mills theory tended to suggest that the $\mathcal{N}^{(m)}$ should only exist for a finite number of values of $m$.

While there is a reasonably simple obstruction theory [E-L] allowing one, in principle, to analyze the problem of extending such 'thickenings" $\mathscr{N}^{(m)}$ from one value of $m$ to the next, a direct assault on the problem foundered on the rocks of the Penrose transform - the relevant cohomology groups turn out to be sufficiently complicated so as to make the extension problem seem rather opaque. The daring idea of Baston and Mason [B-M] was to exploit the fact that the transition functions of $\mathscr{N}$ may be taken to be contact transformations and one should therefore try to extend them as such. Working with $\mathbf{A} \subset \mathbb{P}(T) \times \mathbb{P}\left(T^{*}\right)$ as their model, they noted that infinitesimal contact transformations are generated by sections of the line bundle $\mathcal{O}(1,1)$ over $\mathbf{A}$, while sections of the same line bundle on $\mathbb{P}(T) \times \mathbb{P}\left(T^{*}\right)$ may be used to generate analogous vector fields by using the symplectic structure of $T \times T^{*}$. In these terms, they were able to provide strong evidence for the following conjecture: curved analogs of $\mathbf{A}^{(4)}$ exist for any ambitwistor space, while the obstruction to the existence of an analog of $\mathbf{A}^{(5)}$ is 
the Bach tensor [B],

$$
B_{a b}=\left(\nabla^{c} \nabla^{d}-\frac{1}{2} R^{c d}\right) C_{a b c d}
$$

where $R_{c d}$ and $C_{a b c d}$ represent, respectively, the Ricci and Weyl curvatures; moreover, the existence of an analog of $\mathbf{A}^{(6)}$ is equivalent to the vanishing of the Eastwood-Dighton tensor [D],

$$
E_{a b c}=\frac{1}{2} C_{{ }_{c}{ }_{b}}^{e f} \nabla^{d}(* C)_{a f e d}-\frac{1}{2}(* C)^{e}{ }_{c}^{f}{ }_{b} \nabla^{d} C_{a f e d},
$$

where ${ }^{*} C$ is the Hodge-star of the Weyl curvature as defined by

$$
\left({ }^{*} C\right)_{\mathrm{abcd}}=\frac{1}{2} \varepsilon_{a b}^{e f} C_{e f c d} .
$$

It is a remarkable fact $[\mathrm{B}-\mathrm{M}, \mathrm{K}-\mathrm{N}-\mathrm{T}]$ that, when the Weyl curvature is algebraically general, the vanishing of these tensors is equivalent to the metric being conformal to an Einstein metric; moreover, these tensors vanish on any Einstein space as a consequence of the Bianchi identities.

The present investigation properly begins with the following question: what structure on $\mathbb{P}(T) \times \mathbb{P}\left(T^{*}\right)$ reflects the face that $T \times T^{*}$ has a natural symplectic structure? The answer is the bivector field

$$
\tau=\pi_{*}\left(\left(Z^{j} W_{j}\right) \frac{\partial}{\partial Z^{k}} \wedge \frac{\partial}{\partial W_{k}}\right)
$$

where

$$
\pi:(T-\{0\}) \times\left(T^{*}-\{0\}\right) \rightarrow \mathbb{P}(T) \times \mathbb{P}\left(T^{*}\right)
$$

is the tautological projection. On $\left[\mathbb{P}(T) \times \mathbb{P}\left(T^{*}\right)\right]-\mathbf{A}, \tau$ is the inverse of the symplectic form of the manifold obtained from $Z\lrcorner W=1$ as the MarsdenWeinstein symplectic quotient. This tensor $\tau$ gives $\mathbb{P}(T) \times \mathbb{P}\left(T^{*}\right)$ a Poisson structure [W], meaning that the Poisson bracket

$$
\{f, g\}=\tau(d f, d g)
$$

equips the holomorphic functions on $\mathbb{P}\left(T^{*}\right) \times \mathbb{P}\left(T^{*}\right)$ with the structure of a sheaf of Lie algebras over $\mathbb{C}$. This suggests that, rather than just looking for thickenings of $\mathscr{N}$, one should look for thickenings equipped with Poisson structures. We proceed by first making this precise (Sects. 1-2), setting up an obstruction theory (Sect. 3), and, in Sect. 4, relating these constructions to the first-order thickening defined in [L1]. In Sects. 5-6, we show that, in the ambitwistor context, the Poisson thickening problem is equivalent to the ordinary thickening problem, while in the process producing the third order thickening $\mathscr{N}^{(3)}$ of an arbitrary such space. In Sects. 7-9 we compute the obstruction to higher order thickening by "localizing" the problem to the infinitesimal neighborhoods of a sky, thereby proving the conjectures of Baston and Mason. In Sect. 10 we then describe some relations between this work and conformal supergravity, suggesting an alternate approach via complex supermanifolds, and point out a number of directions in which one might seek extensions of the present results. 


\section{Poisson Thickenings}

Let $X$ be a complex $(2 n+1)$-manifold. A complex contact structure on $X$ is a rank $2 n$ holomorphic sub-bundle $D$ of the tangent bundle of $X$ such that the skew form

$$
\begin{aligned}
A: D \times D & \rightarrow T X / D \\
(v, w) & \mapsto[v, w] \bmod D
\end{aligned}
$$

is non-degenerate. Setting $L=T X / D$ and letting $\theta \in \Gamma\left(X, \Omega^{1}(L)\right)$ denote the canonical projection $T X \rightarrow L$, the latter condition is equivalent to

$$
\theta \wedge(d \theta)^{\wedge n} \neq 0,
$$

so that in particular we have an isomorphism $L^{\otimes(n+1)} \cong \kappa^{-1}$, where $\kappa$ is the canonical line bundle of $X: \mathcal{O}(\kappa):=\Omega^{2 n+1}$. Darboux's theorem (cf. [A]) states that, for each local trivialization of $L$, the corresponding 1 -form $\vartheta$ may be expressed as

$$
\vartheta=d q^{0}+\sum_{j=1}^{n} p_{j} d q^{j}
$$

in terms of suitable local holomorphic coordinates $\left(q^{0}, \ldots, q^{n}, p_{1}, \ldots, p_{n}\right)$ on $X$.

Associated with an arbitrary complex contact manifold is a complex symplectic manifold. Indeed, the adjoint of the canonical projection $T X \rightarrow L$ is an inclusion $L^{*} \rightarrow T^{*} X$; this realizes $L^{*}$ as the annihilator of $D \subset T X$. But $T^{*} X$ carries a symplectic form $\omega \in \Gamma\left(T^{*} X, \Omega^{2}\right)$, and this 2 -form restricts to $L^{*}-0_{L^{*}}$ to yield a closed 2-form $\omega$. Requiring that $\omega$ be symplectic is equivalent to the requirement that $\theta$ be a contact structure - i.e. that $A=\left.d \theta\right|_{D}$ be non-degenerate. Indeed, assuming that $D$ is a contact structure, taking a local trivialization of $L$ and letting $s$ be the corresponding fiber coordinate for $L^{*}$ we have

$$
\omega=d(s \vartheta)=d s \wedge d q^{0}+\sum_{j=1}^{n} d\left(s p_{j}\right) \wedge d q^{j} .
$$

Notice, incidentally, that $\omega$ drops rank at the zero section $0_{L^{*}} \subset L^{*}$.

Now for any holomorphic line bundle $L$ there is a canonical biholomorphism

$$
\Phi: L-0_{L}-L^{*}-0_{L^{*}}
$$

characterized by $\langle\Phi(\lambda), \lambda\rangle \equiv 1$; in each fiber, $\Phi$ is an inversion of the punctured plane, but the morphism $\Phi$ is natural in the sense that it is an isomorphism of principal $(\mathbb{C}-\{0\})$-bundles, corresponding to the familiar operation of passing from a frame to its dual frame. In the present case, we therefore get a symplectic form on $L-0_{L}$ given by $\Phi^{*} \omega$. But unlike the 2 -form on $L^{*}-0_{L^{*}}$, this form is singular at the zero section. Indeed, introducing a fiber coordinate $t=s^{-1}$ on $L$ and using adapted coordinates $\left(q^{0}, \ldots, q^{n}, p_{1}, \ldots, p_{n}\right)$ on $X$ as before,

$$
\begin{aligned}
\Phi^{*} \omega & =d\left(\frac{\vartheta}{t}\right) \\
& =-\frac{1}{t^{2}} d t \wedge\left(d q^{0}+\Sigma p_{j} d q^{j}\right)+\frac{1}{t} \Sigma d p_{j} \wedge d q^{j} .
\end{aligned}
$$


However, the bivector field $\tau=\left(\Phi^{*} \omega\right)^{-1} \in \Gamma\left(L, \wedge^{2} T L\right)$ extends holomorphically across the zero section:

$$
\tau=t\left[\left(t \frac{\partial}{\partial t}+\Sigma p_{j} \frac{\partial}{\partial p_{j}}\right) \wedge \frac{\partial}{\partial q^{0}}+\Sigma \frac{\partial}{\partial q^{j}} \wedge \frac{\partial}{\partial p_{j}}\right]
$$

This gives $L$ the structure of a Poisson manifold (cf. [W]), in that, defining the Poisson bracket

by

$$
\{,\}: \mathcal{O} \times \mathcal{O} \rightarrow \mathcal{O}
$$

the Jacobi identity

$$
\{f, g\}=\tau(d f, d g)
$$

$$
\{f,\{g, h\}\}+\{g,\{h, f\}\}+\{h,\{f, g\}\}=0
$$

is satisfied, making the holomorphic functions $\mathcal{O}$ on $L$ into a sheaf of Lie algebras. The tensor field $\tau$ defining this Poisson structure will be called the exelissic form or the cosymplectic form. (Exelissic < Gr. "unwinding"; cf. symplectic < Gr. "intertwining".)

Let $\mathscr{T} \subset \mathcal{O}$ denote the ideal of $X=0_{L} \subset L$. By direct inspection of $(*)$, we notice that our Poisson bracket satisfies

$$
\left\{\mathscr{T}^{k}, \mathscr{T}^{l}\right\} \subset \mathscr{T}^{k+l+1} .
$$

If we define

$$
\mathcal{O}_{m}:=\mathcal{O} / \mathscr{T}^{m+1}
$$

it therefore follows that $\{$,$\} gives \mathcal{O}_{m}$ the structure of a sheaf of nilpotent Lie algebras. Moreover, since the constants $\mathbb{C}$ are in the center of $\mathcal{O}_{m}, \mathcal{O}_{m} / \mathbb{C}$ becomes a sheaf $\mathscr{A}_{m+1}$ of nilpotent Lie algebras, $m=0,1,2, \ldots$. We define

$$
\mathscr{G}_{m}:=\exp \mathscr{A}_{m},
$$

thereby obtaining a sheaf of nilpotent Lie groups. Concretely, $\mathscr{G}_{m}$ may be identified with $\mathcal{O}_{m-1} / \mathbb{C}$ equipped with a multiplicative structure given by the BakerCampbell-Hausdorff formula

$$
f \cdot g:=f+g+\frac{1}{2}\{f, g\}+\frac{1}{12}\{f-g,\{f, g\}\}+\cdots .
$$

The significance of this sheaf of groups is as follows: there is a natural injective map $\mathcal{O}_{m-1} / \mathbb{C} \rightarrow \operatorname{Der}\left(\mathcal{O}_{m}\right)$ given by $f \mapsto\{f, \cdot\}$, and this realizes $\mathscr{A}_{m}$ as a nilpotent subalgebra of $\operatorname{Der}\left(\mathcal{O}_{m}\right)$. Therefore $\mathscr{G}_{m}$ is a certain nilpotent subgroup of Aut $\left(\mathcal{O}_{m}\right)$.

Now elements of $H^{1}\left(\operatorname{Aut}\left(\mathcal{O}_{m}\right)\right)$ are precisely isomorphism classes of thickenings [E-L] of $X$, meaning ringed spaces locally isomorphic to $\left(X, \mathcal{O}_{m}\right)$; such creatures are to be thought of as abstract versions of $m$-jets of codimension 1 embeddings of $X$. In particular, since $\mathscr{G}_{m} \subset \operatorname{Aut}\left(\mathcal{O}_{m}\right)$, elements of $H^{1}\left(\mathscr{G}_{m}\right)$ may be interpreted as thickenings.

Definition. Let $(X, D)$ be a complex contact manifold. A thickening of $X$ of order $m$ is said to be of Poisson type if its isomorphism class is in the image of

$$
H^{1}\left(X, \mathscr{G}_{m}\right) \rightarrow H^{1}\left(X, \operatorname{Aut}\left(\mathcal{O}_{m}\right)\right) \text {. }
$$

An element of $H^{1}\left(X, \mathscr{G}_{m}\right)$ will be called an $m^{\text {th }}$ order Poisson thickening of $X$. 
While this definition is sufficient for technical purposes, it perhaps does not hold much appeal for the intuition. In order to therefore provide the reader with some mental images to accompany the subsequent discussion, a geometrical picture of a Poisson thickening will now be indicated without proof.

The elements of $\mathscr{G}_{m}$ act in a manner preserving the exelissic form $\tau$, so an element of $H^{1}\left(\mathscr{G}_{m}\right)$ gives rise to a thickening which carries such a form. Elements of $\mathscr{G}_{m}$ also preserve the subspace

$$
D \oplus L \subset T X \oplus L
$$

hence the extended tangent bundle $\hat{T} X$ of a Poisson thickening contains a codimension 1 sub-bundle $\hat{D}$ with $D=\hat{D} \cap T X$. Finally, a Poisson thickening carries a divisor line-bundle with Lie algebra structure; if $\mathscr{L} \rightarrow L$ denotes the divisor line-bundle of $0_{L} \subset L$, and if $\mathscr{L}_{(m)}:=\mathscr{L} \otimes \mathcal{O}_{m}$, then the Poisson bracket extends to a Lie-algebra multiplication

$$
\{,\}: \mathscr{L}_{(m)} \times \mathscr{L}_{(m)} \rightarrow \mathscr{L}_{(m)},
$$

and $\mathscr{G}_{m}$ acts on $\mathscr{L}_{(m)}$ by Poisson brackets, thus giving birth to a divisor line bundle with Poisson bracket over the thickening associated to any element of $H^{1}\left(X, \mathscr{G}_{m}\right)$. Rather intriguingly, this new Lie algebra is an extension the infinitesimal contact transformations of $(X, D)$ by $\left(\mathcal{O}_{(m-1)} / \mathbb{C},\{\},\right)$.

One can check that these structures characterize a Poisson thickening. This formulation seems so unwidely, however, that we will never actually use it, for which reason the details have been left to the imagination of the interested reader.

\section{The Extension Problem}

Let $(X, D)$ be an arbitrary complex contact manifold, and let $E \rightarrow X$ be any holomorphic line bundle. The isomorphism class of $E$ is an element of $H^{1}\left(X, \mathcal{O}_{*}\right)$, where $\mathcal{O}_{*}$ is the multiplicative sheaf of non-zero holomorphic functions, and hence give rise to an element of $H^{1}(X, \mathcal{O} / \mathbb{C})$ via the epimorphism

$$
\log : \mathcal{O}_{*} \rightarrow \mathcal{O} / \mathbb{C}
$$

Since $\mathscr{G}_{1}=\mathscr{O} / \mathbb{C}$ with its standard group structure, this means that the line bundle $E$ gives rise to a first-order Poisson thickening of $X$. One may then ask whether this thickening extends to higher orders; for $E$ non-trivial, this extension problem will generally be obstructed.

We now specialize to the case of $X=\mathscr{N}$, where $\mathcal{N}$ is the ambitwistor space (i.e. the space of null geodesics) of a complex-Riemannian 4-manifold ( $\mathscr{M}, \mathrm{g})$. Such an ambitwistor space carries a unique complex contact structure [L2], and the complex structure of $\mathscr{N}$ completely encodes the conformal structure of $(\mathscr{M}, \mathfrak{g})$. Let us assume that $(\mathscr{M}, \mathfrak{g})$ has a spin structure, so that there are rank 2 vector bundles $\mathbf{S}_{ \pm} \rightarrow \mathscr{M}$ with

$$
\mathfrak{S}_{+} \otimes \mathbb{S}_{-} \cong T \mathscr{M}
$$

in a manner such that the simple tensor products of spinors correspond to null vectors, and such that $\Lambda^{2} \mathbb{S}_{+} \cong \Lambda^{2} \mathbb{S}_{-}$in a canonical fashion. Assuming that the null geodesics of $\mathscr{M}$ are all simply connected, we may then define line bundles 
$L_{ \pm} \rightarrow \mathscr{N}$ by

$$
\left(L_{ \pm}^{*}\right)_{\gamma}=\left\{\sigma \in \Gamma\left(\gamma, \mathcal{O}\left(\left.\mathbf{S}_{ \pm}^{*}\right|_{\gamma}\right)\right) \mid D \sigma \equiv 0 \text { and } \sigma \cdot(T \gamma)=0\right\},
$$

where $D$ is covariant differentiation along $\gamma$; explicitly, $L_{+}$is dual to the solution spaces of

$$
\lambda^{A} \pi^{A^{\prime}} \nabla_{A A^{\prime}} \lambda_{B}=0
$$

and $L_{-}$is dual to the solution spaces of

$$
\lambda^{A} \pi^{A^{\prime}} \nabla_{A A^{\prime}} \pi_{B^{\prime}}=0
$$

These line bundles are conformally invariant and satisfy $L_{+} \otimes L_{-}=L$.

Now $L_{+} \rightarrow \mathcal{N}$ defines a first-order Poisson thickening of $\mathscr{N}$ by the general mechanism defined above. This will be called the canonical first order thickening of $\mathscr{N}$.

This paper has as its aim the proof of the following result, which will be proved in subsequent sections.

Main Theorem. The canonical first order thickening of $\mathcal{N}$ always extends uniquely to a fourth order Poisson thickening. It can be extended to a fifth order Poisson thickening iff the Bach tensor

$$
B_{a b}=\left(\nabla^{c} \nabla^{d}-\frac{1}{2} R^{c d}\right) C_{a b c d}
$$

of $(\mathscr{M}, \mathfrak{g})$ vanishes. It can be extended to sixth order iff, in addition, the EastwoodDighton tensor

$$
E_{a b c}=\Psi_{A B C D} \nabla^{D D^{\prime}} \tilde{\Psi}_{A^{\prime} B^{\prime} C^{\prime} D^{\prime}}-\tilde{\Psi}_{A^{\prime} B^{\prime} C^{\prime} D^{\prime}} \nabla^{D D^{\prime}} \Psi_{A B C D}
$$

vanishes.

Remark. If the Weyl tensor is algebraically general, these two conditions imply [B-M] that the metric $\mathfrak{g}$ is conformal to Einstein. On the other hand [L4], thickenings of all orders exist if the Weyl curvature is self-dual or anti-self-dual.

\section{Obstruction Theory}

Given an order $m$ Poisson thickening of a complex contact manifold $(X, D)$, when can one extend it to a Poisson thickening of order $m+1$ ? If this can be done, how many different extensions are possible?

Proposition 3.1. The obstruction to extending a Poisson thickening of order $m \geqq 1$ to order $m+1$ is an element of $H^{2}\left(X, \mathcal{O}\left(L^{-m}\right)\right)$. If such an extension exists, the set of isomorphism classes of such extensions is acted on transitively by

$$
H^{1}\left(X, \mathcal{O}\left(L^{-m}\right)\right)
$$

Proof. We have short exact sequences

$$
\mathcal{O}\left(L^{-m}\right) \mapsto \mathscr{G}_{m+1} \rightarrow \mathscr{G}_{m}
$$

of sheaves of groups, where $\mathcal{O}\left(L^{-1}\right)$ is contained in the center of $\mathscr{G}_{m+1}$. Hence the non-abelian version $[E-L, M]$ of the long exact sequence says that we have an 
exact sequence

$$
H^{1}\left(\mathcal{O}\left(L^{-m}\right)\right) \rightarrow H^{1}\left(\mathscr{G}_{m+1}\right) \rightarrow H^{1}\left(\mathscr{G}_{m}\right) \rightarrow H^{2}\left(\mathcal{O}\left(L^{-m}\right)\right) \text {. Q.E.D. }
$$

Remark. The $H^{0}$ piece of the "long exact sequence" is more complicated than in the abelian case, even for our present central extension. For a given thickening $t \in H^{1}\left(\mathscr{G}_{m}\right)$, one creates a new sheaf of groups $t \mathscr{G}_{m} t^{-1}$ whose global sections form the isotropy group of the action of $H^{1}\left(\mathcal{O}\left(L^{-m}\right)\right)$ on the kernel of $H^{1}\left(\mathscr{G}_{m+1}\right) \rightarrow H^{1}\left(\mathscr{G}_{m}\right)$. This isotropy group $H^{0}\left(t \mathscr{G}_{m} t^{-1}\right)$ is thus exactly given by $\left.\exp (\tau\lrcorner d\left(H^{0}\left(\mathcal{O}_{(m-1)} / \mathbb{C}\right)\right)\right)$, where $\tau$ is the exelissic form of the Poisson thickening $t$ and where $\mathcal{O}_{(m-1)}$ is the structure sheaf of the associated thickening of order $m-1$. Thus $H^{1}\left(\mathcal{O}\left(L^{-m}\right)\right)$ will act freely on choices of extension provided that $H^{0}(\mathcal{O} / \mathbb{C})=H^{0}\left(\mathcal{O}\left(L^{-1}\right)\right)=\cdots$ $=H^{0}\left(\mathcal{O}\left(L^{-m+1}\right)\right)=0$. In the case of interest, these vanishing statements will hold, and the action is actually free.

We now specialize to the case of $X=\mathscr{N}$, an ambitwistor space. By applying the Penrose transform, we obtain the following table of cohomology groups $H^{p}\left(\mathcal{N}, \mathcal{O}\left(L^{-m}\right)\right)$ under the assumption that $\mathscr{M}$ is Stein and that the null geodesics of $\mathscr{M}$ are all contractible, as will, for example, be the case if $\mathscr{M}$ is geodesically convex. (Here the numbers in the square brackets are conformal weights, while $\odot_{0}$ denotes the trace-free symmetric tensor product. Sheaves on $\mathscr{M}$ are used as short-hand for their global sections, thereby saving space in the table.)

Table 1. $H^{p}\left(\mathscr{N}, \mathcal{O}\left(L^{-m}\right)\right)$

\begin{tabular}{llll}
\hline$m \backslash p$ & 0 & 1 & 2 \\
\hline 0 & $\mathbb{C}$ & $\Omega^{1} / d \Omega^{0}$ & 0 \\
1 & 0 & $\mathcal{O}[-2]$ & 0 \\
2 & 0 & 0 & $\mathcal{O}[-2]$ \\
3 & 0 & 0 & $\operatorname{Ker} d: \Omega^{3} \rightarrow \Omega^{4}$ \\
4 & 0 & 0 & $\operatorname{Ker} \operatorname{div}: \odot_{0}^{2} \Omega^{1}[-2] \rightarrow \Omega^{1}[-4]$ \\
5 & 0 & 0 & $\operatorname{Ker} \operatorname{div}: \bigodot_{0}^{3} \Omega^{1}[-2] \rightarrow \odot_{0}^{2} \Omega^{1}[-4]$ \\
\hline
\end{tabular}

Let us recall how these answers are arrived at. There is a double fibration

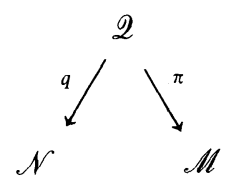

where $\pi: \mathscr{Q} \rightarrow \mathscr{M}$ is the bundle of projective null vectors and where $q: \mathscr{Q} \rightarrow \mathcal{N}$ sends a null direction to the null geodesic to which it is tangent. (Thus $\pi$ has typical fiber $\mathbb{P}_{1} \times \mathbb{P}_{1}$, and each fiber of $q$ is a contractible complex curve.) By a theorem of Buchdahl $[\mathrm{Bu}]$,

$$
q^{-1}: H^{*}\left(\mathcal{N}, \mathcal{O}\left(L^{-m}\right)\right) \cong H^{*}\left(\mathscr{Q}, q^{-1} \mathcal{O}\left(L^{-m}\right)\right)
$$

whereas the relative holomorphic deR ham sequence on $\mathscr{Q}$ is the short exact sequence

$$
0 \rightarrow q^{-1} \mathcal{O}\left(L^{-m}\right) \rightarrow \mathcal{O}(-m,-m) \underset{d_{q}}{\rightarrow} \mathcal{O}(-m+1,-m+1)[2] \rightarrow 0,
$$


where $\mathcal{O}(-1,-1) \rightarrow \mathscr{Q}$ is the tautological line bundle with conformal weight [ -2$]$, and where $d_{q}$ is differentiation up the fibers of $q$. The entries in Table 1 are then read off from the associated long exact sequence, using the LeRay spectral sequence and the fact that $\mathscr{M}$ is Stein to identify the cohomology of sheaves on $\mathscr{Q}$ with sections of the corresponding direct image sheaves on $\mathscr{M}$.

Now the above argument tells us how to understand the cohomology of $\mathscr{N}$ explicitly. In particular, an element of $H^{2}\left(\mathscr{N}, \mathcal{O}\left(L^{-m}\right)\right)$ vanishes iff, for each $x \in \mathscr{M}$, its image under the restriction map

$$
H^{2}\left(\mathscr{N}, \mathcal{O}\left(L^{-m}\right)\right) \rightarrow H^{2}\left(Q_{x}, \mathcal{O}\left(L^{-m}\right)\right)
$$

vanishes. By contrast, the restriction maps

$$
H^{1}\left(\mathcal{N}, \mathcal{O}\left(L^{-1}\right)\right) \rightarrow H^{1}\left(Q_{x}, \mathcal{O}\left(L^{-1}\right)\right)
$$

are always zero, and it is the first derivative maps

$$
H^{1}\left(\mathcal{N}, \mathcal{O}\left(L^{-1}\right)\right) \rightarrow H^{1}\left(Q_{x}, \mathcal{O}\left(N^{*} \otimes L^{-1}\right)\right),
$$

where $N^{*} \rightarrow Q_{x}$ is the conormal bundle, that characterizes a cohomology class; i.e. and element of $H^{1}\left(\mathscr{N}, \mathcal{O}\left(L^{-1}\right)\right)$ is zero iff its restriction to the first infinitesimal neighborhood of each sky $Q_{x} \subset \mathscr{N}$ vanishes.

Now one of the key results of this paper will be that the canonical first-order Poisson thickening always extends uniquely to fourth order (Theorem 9.1). This entails showing that the freedom

$$
H^{1}\left(\mathscr{N}, \mathcal{O}\left(L^{-1}\right)\right)=\Gamma(\mathscr{M}, \mathcal{O}[-2])
$$

in extending to second order is exactly cancelled by the obstruction, in

$$
H^{2}\left(\mathscr{N}, \mathcal{O}\left(L^{-2}\right)\right)=\Gamma(\mathscr{M}, \mathcal{O}[-2])
$$

to extending to third order. We will do this by "localizing" the question to an infinitesimal neighborhood of an arbitrary sky $Q_{x} \subset \mathscr{N}$, using the above observation that the relevant cohomology is indeed "locally determined." The same technique will be used to analyze the higher order obstructions.

Before we can embark on this analysis, however, we will need to understand a bit more about the canonical first-order Poisson thickening $\mathscr{N}^{(1)}$, and, in particular, study the normal bundle of a sky $Q_{x}$ relative to $\mathscr{N}^{(1)}$. After doing this in the next section, we will be in a position to return to the detailed analysis of the obstruction.

\section{A Closer Look at First Order Thickenings}

Recall [E-L] that a first order thickening of any complex manifold $X$ is completely characterized by its extended tangent bundle $\hat{T} X \rightarrow X$ and by the inclusion $T X \subset \hat{T} X$. In this section we will give a direct construction of the extended tangent bundle of a Poisson thickening arising from a holomorphic line bundle $E \rightarrow X$ over a complex contact manifold via

$$
\log _{*}: H^{1}\left(X, \mathcal{O}_{*}\right) \rightarrow H^{1}(X, \mathcal{O} / \mathbb{C})=H^{1}\left(X, \mathscr{G}_{1}\right) .
$$


We begin by constructing an extension

$$
0 \rightarrow D \rightarrow \hat{D} \rightarrow L \rightarrow 0
$$

of the contact line bundle $L=T X / D$ by the contact distribution $D$. To do so, consider the jet sequence

$$
0 \rightarrow\left(T^{*} X \otimes E\right) \rightarrow J^{1} E \rightarrow E \rightarrow 0
$$

of our given line bundle. Let us $\bmod$ out by $L^{*} \subset T^{*} X$ :

$$
0 \rightarrow D^{*} \otimes E \rightarrow\left(J^{1} E\right) /\left(L^{*} \otimes E\right) \rightarrow E \rightarrow 0 .
$$

Twisting by $L \otimes E^{*}$ we have

$$
0 \rightarrow D^{*} \otimes L \rightarrow\left(L \otimes E^{*} \otimes J^{1} E\right) / \mathcal{O} \rightarrow L \rightarrow 0 .
$$

But $A=\left.d \theta\right|_{D} \in \Gamma\left(X,\left(\Lambda^{2} D^{*}\right) \otimes L\right)$ gives an isomorphism $D \cong D^{*} \otimes L$, so this sequence becomes

$$
0 \rightarrow D \stackrel{j_{1}}{\rightarrow}\left(L \otimes E^{*} \otimes J^{1} E\right) / \mathcal{O} \stackrel{\pi_{1}}{\rightarrow} L \rightarrow 0 .
$$

This is the promised preliminary extension.

To produce the extended tangent bundle $\hat{T} X$, one remembers that we also have the tautological extension

$$
0 \rightarrow D \stackrel{J_{2}}{\rightarrow} T X \stackrel{\pi_{2}}{\rightarrow} L \rightarrow 0
$$

by which to "push out" the previous extension, meaning that we wish to have the diagram

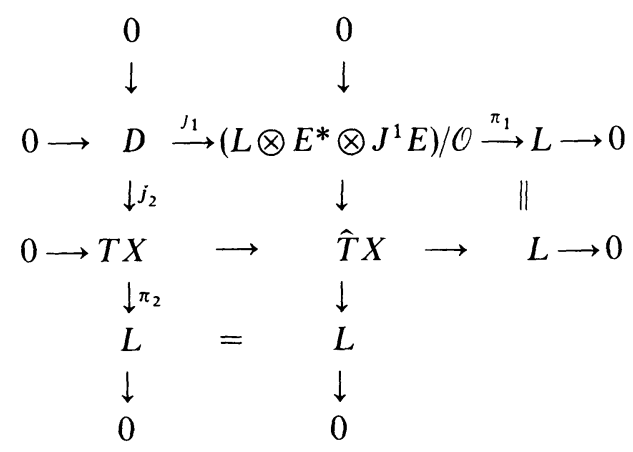

with rows and columns exact. This can be done uniquely up to isomorphism by letting $\hat{T} X$ defined to be quotient of $T X \oplus\left[\left(L \oplus E^{*} \oplus J^{1} E\right) / \mathcal{O}\right]$ by the image of $D$ under $j_{1} \otimes\left(-j_{2}\right)$.

Now the bundle $\hat{T} X$ constructed in this way coincides with the extended tangent bundle of the Poisson thickening associated to $[E] \in H^{1}\left(X, \mathcal{O}_{*}\right)$ by applying $\log _{*}$. To see this, recall that the extension class of

$$
0 \rightarrow T^{*} X \rightarrow E^{*} \otimes J^{1} E \rightarrow \mathcal{O} \rightarrow 0
$$

is given by $\left[d \log f_{\alpha \beta}\right] \in H^{1}\left(X, \Omega^{1}\right)$, where $\left\{f_{\alpha \beta}\right\}$ is a $\hat{C} e c h$ cocycle for $[E] \in H^{1}\left(X, \mathcal{O}_{*}\right)$. 
Restricting $d \log f_{\alpha \beta}$ to $D$ and identifying $D^{*}$ with $D \otimes L^{*}$ via the inverse of $\left.d \theta\right|_{D}$ precisely corresponds to contracting $d \log f_{\alpha \beta}$ with the exelissic form $\tau$.

We now apply this observation to ambitwistor spaces.

Proposition 4.1. The canonical first-order Poisson thickening of an ambitwistor space is the same as the first order thickening described in [L1].

Proof. The extended tangent bundle $\widetilde{T}_{\gamma} \mathcal{N}$ of the latter thickening at $\gamma \in \mathscr{N}$ is defined to be the solutions of the generalized Jacobi equation

$$
\mathscr{D}^{2}\left[\begin{array}{c}
\omega^{A} \\
\xi^{A^{\prime}}
\end{array}\right]=-\left[\begin{array}{cccc}
\Phi_{B} \lambda^{A} & \vdots & \Psi_{B^{\prime}} \cdot \lambda^{A} \\
\dot{\Psi}_{B} \cdot \pi^{A^{\prime}} & \vdots & \dot{\Phi}_{B^{\prime}} \cdot \pi^{A^{\prime}}
\end{array}\right]\left[\begin{array}{c}
\omega^{B} \\
\xi^{B^{\prime}}
\end{array}\right]
$$

along the null geodesic $\gamma \subset \mathscr{M}$, subject to the added constraint that

$$
\mathscr{D}\left[\begin{array}{c}
\lambda_{A} \omega^{A} \\
\pi_{A^{\prime}} \xi^{A^{\prime}}
\end{array}\right]=0
$$

Here $X^{A A^{\prime}}=\lambda^{A} \pi^{A^{\prime}}$ is tangent to $\gamma, \mathscr{D}=\nabla_{X}, \mathscr{D} \lambda=\mathscr{D} \pi=0$, while

and

$$
\begin{aligned}
& \Phi_{A}:=\Phi_{A B A^{\prime} B^{B}} \lambda^{B} \pi^{A^{\prime}} \pi^{B^{\prime}}, \\
& \Psi_{A}:=\Psi_{A B C D} \lambda^{B} \lambda^{C} \lambda^{D},
\end{aligned}
$$$$
\Phi_{A^{\prime}}:=\Phi_{A B A^{\prime} B^{\prime}} \lambda^{A} \lambda^{B} \pi^{B^{\prime}},
$$$$
\widetilde{\Psi}_{A^{\prime}}:=\widetilde{\Psi}_{A^{\prime} B^{\prime} C^{\prime} D^{\prime}} \pi^{B^{\prime}} \pi^{C^{\prime}} \pi^{D^{\prime}}
$$

are curvature components. Moreover, we take $\omega$ and $\xi$ to be homogeneous with respect to $\lambda$ and $\pi$ by requiring that under the transformation

$$
\lambda \mapsto C \lambda, \quad \pi \mapsto \tilde{C} \lambda,
$$

we have

$$
\omega \mapsto \tilde{C} \omega, \quad \xi \mapsto C \xi .
$$

The natural inclusion $T \mathscr{N} \hookrightarrow \hat{T} \mathcal{N}$ is then given by sending a Jacobi field $J$ to the pair

$$
\left[\begin{array}{c}
\omega^{A} \\
\xi^{A^{\prime}}
\end{array}\right]=\left[\begin{array}{c}
J^{A A^{\prime}} \pi_{A^{\prime}} \\
J^{A A^{\prime}} \lambda_{A}
\end{array}\right]
$$

Now consider the subspace $\tilde{D}$ of the solution space consisting of pairs such that $\xi^{A^{\prime}} \pi_{A^{\prime}}=0$; from the constraint it follows that this holds identically if it holds at any point of $\gamma$. Then $\widetilde{D} \subset \widetilde{T} \mathcal{N}$ has codimension 1 and

$$
\tilde{T} \mathcal{N}=T \mathscr{N}+\tilde{\mathscr{D}} \text {. }
$$

Moreover, $D \subset \tilde{D}$ and we have an exact sequence

$$
0 \rightarrow D \rightarrow \underset{w}{\rightarrow} L \rightarrow 0
$$

where $\varpi$ is given by

$$
\varpi\left(\left[\begin{array}{c}
\omega^{A} \\
\xi^{A^{\prime}}
\end{array}\right]\right)=\omega^{A} \lambda_{A}
$$


It suffices to show that this extension is canonically isomorphic to

$$
0 \rightarrow D^{*} \otimes L \rightarrow\left(L_{-} \otimes J^{1} L_{+}\right) / \mathcal{O} \rightarrow L \rightarrow 0 .
$$

To see this, let us first consider the tangent space of the total space of $L_{+} \rightarrow \mathscr{N}$. At a base point represented by $\left(\gamma, \lambda_{A}\right)$, where as before $\mathscr{D} \lambda=0$ along $\gamma$, consider the subspace $\mathfrak{D} \subset T L_{+}$which is the inverse image of $D \subset T \mathcal{N}$. This hyperplane may be identified [L1] with the solution space of the system

$$
\begin{aligned}
\mathscr{D}^{2}\left[\begin{array}{l}
\alpha \\
\beta
\end{array}\right] & =-\left[\begin{array}{ll}
\Phi & \tilde{\Psi} \\
\Psi & \Phi
\end{array}\right]\left[\begin{array}{l}
\alpha \\
\beta
\end{array}\right], \\
\mathscr{D} \mu_{A} & =\alpha \Psi_{A}+\beta \Phi_{A}
\end{aligned}
$$

subject to the constraint that

$$
\mathscr{D} \beta=-\lambda^{A} \mu_{A} .
$$

Here $\Phi=\Phi_{A^{\prime}} \pi^{A^{\prime}}=\Phi_{A} \lambda^{A}, \Psi=\Psi_{A} \lambda^{A}$, and $\widetilde{\Psi}=\widetilde{\Psi}_{A^{\prime}} \pi^{A^{\prime}}$. (If $\gamma(s)$ is a family of affinely parameterized null geodesics such that $\mathrm{g}\left(\frac{\partial \gamma}{\gamma s}, \frac{\partial \gamma}{\partial s}\right)=0$, and if $\lambda(s)$ is a family of adapted spinors which are parallel along this family, then the tangent of this curve in $L_{+}^{*}$ is represented by $\left(\alpha, \beta, \mu_{A}\right)$, as defined by

and

$$
\begin{aligned}
\alpha \lambda^{A} & =J^{A A^{\prime}} \pi_{A^{\prime}}, \\
\beta \pi^{A^{\prime}} & =J^{A A^{\prime}} \lambda_{A^{\prime}}, \\
\mu_{A} & =\frac{\nabla}{\partial s}\left(\lambda_{A}\right),
\end{aligned}
$$

where $J=\frac{\partial \gamma}{\partial s}$.) But since sections of $L_{+}$correspond to functions on the total space of $L_{+}^{*}$ of homogeneity $1, J^{1} L_{+}$may be obtained from $T^{*}\left(L^{*}\right)$ :

$$
J^{1} L_{+}=\left(T^{*}\left(L^{*}+-0_{L_{+}^{*}}\right)\right) / \mathbb{C}-\{0\},
$$

where the action of $\mathbb{C}-\{0\}$ combines the pull-back under scalar multiplication maps with scalar multiplication in the cotangent space. Hence

$$
L_{-} \otimes J^{1} L_{+} / \mathcal{O}=\left(\mathfrak{D}^{*} / \mathbb{C}-\{0\}\right) \otimes L_{-},
$$

where the trivial line bundle $\mathcal{O} \subset L_{-} \otimes J^{1} L_{+}$is spanned by the contact form.

Now notice that there is a homogeneous pairing between $\mathfrak{D}$ and $\hat{D}$. For if

$$
\mathscr{D}^{2}\left[\begin{array}{c}
\omega^{A} \\
\xi^{A^{\prime}}
\end{array}\right]=-\left[\begin{array}{ccc}
\Phi_{B} \lambda^{A} & \vdots & \widetilde{\Psi}_{B^{\prime}} \lambda^{A} \\
\ddot{\Psi}_{B} \cdot \pi^{A^{\prime}} & \vdots & \ddot{\Phi}_{B^{\prime}} \\
\pi^{A^{\prime}}
\end{array}\right]\left[\begin{array}{c}
\omega^{B} \\
\xi^{B^{\prime}}
\end{array}\right],
$$

with $\xi^{A^{\prime}} \pi_{A^{\prime}}=0$, and $\left(\mathscr{D} \omega^{A}\right) \lambda_{A}=0$, and if

$$
\mathscr{D}^{2}\left[\begin{array}{l}
\alpha \\
\beta
\end{array}\right]=-\left[\begin{array}{ll}
\Phi & \tilde{\Psi} \\
\Psi & \Phi
\end{array}\right]\left[\begin{array}{l}
\alpha \\
\beta
\end{array}\right],
$$

with $\mathscr{D} \mu_{A}=\alpha \Psi_{A}+\beta \Phi_{A}$, and $\mathscr{D} \beta=-\lambda^{A} \lambda_{A}$, then, setting $\xi^{A^{\prime}}=b \pi^{A^{\prime}}$ and $\mathscr{D} \omega^{A}=a \lambda^{A}$, 
it follows that the expression

$$
\Xi\left(\left[\begin{array}{c}
\omega \\
\xi
\end{array}\right], \quad\left[\begin{array}{l}
\alpha \\
\beta \\
\mu
\end{array}\right]\right)=\alpha \mathscr{D} b+\omega^{A} \mu_{A}+\beta a-b \mathscr{D} \alpha
$$

is constant along the null geodesic $\gamma$. Indeed,

$$
\begin{aligned}
\mathscr{D}\left(\alpha \mathscr{D} b-b \mathscr{D} \alpha+\omega^{A} \mu_{A}+\beta a\right)= & (\mathscr{D} \alpha)(\mathscr{D} b)-(\mathscr{D} b)(\mathscr{D} \alpha)+\left(\mathscr{D} \omega^{A}\right) \mu_{A} \\
& +(\mathscr{D} \beta) a+\alpha \mathscr{D}^{2} b-b \mathscr{D}^{2} \alpha+\omega^{A} \mathscr{D} \mu_{A}+\beta \mathscr{D} a \\
= & a \lambda^{A} \mu_{A}-a \lambda^{A} \mu_{A}+\alpha\left(-\Psi_{B} \omega^{B}-\Phi_{B^{\prime}} \xi^{B^{\prime}}\right) \\
& -b(-\Phi \alpha-\tilde{\Psi} \beta)+\omega^{A}\left(\alpha \Psi_{A}+\beta \Phi_{A}\right) \\
& +\beta\left(-\Phi_{B} \omega^{B}-\widetilde{\Psi}_{B^{\prime}} \xi^{B^{\prime}}\right) \\
= & -\alpha b \Phi+b \Phi \alpha+b \tilde{\Psi} \beta-\beta b \tilde{\Psi} \\
= & 0 .
\end{aligned}
$$

This $\Xi$ gives a pairing of $\tilde{D}$ and $(\mathfrak{D} / \mathbb{C}-\{0\}) \otimes L_{-}^{*}$, since it has homogeneity $(1,1)$ in $(\lambda, \pi)$. Since this pairing is non-degenerate, it sets up an isomorphism $\tilde{D} \cong$ $\left(L_{-} \otimes J^{1} L_{+}\right) / \mathcal{O}$. Moreover, if one restricts this isomorphism to the elements with $\omega^{A} \lambda_{A}=0$, the image corresponds to elements of $D^{*}$ which are independent of $\mu$, and so induces an isomorphism $D \cong D^{*} \otimes L$ which may easily be verified to coincide with $d \theta$. Thus the extensions

and

$$
0 \rightarrow D \rightarrow \tilde{D} \rightarrow L \rightarrow 0
$$

$$
0 \rightarrow D \rightarrow \hat{D} \rightarrow L \rightarrow 0
$$

are isomorphic, and the claim follows. Q.E.D.

Notice that it follows that our construction is more "ambidextrous" than it looks - the extended tangent bundles of the Poisson thickenings induced by $L_{+}$ and by $L_{-}$are canonically isomorphic, although the induced distributions $\hat{D}$ differ, corresponding as they do the subspaces $\xi^{A^{\prime}} \pi_{A^{\prime}}=0$ or $\omega^{A} \lambda_{A}=0$, respectively. This is symptomatic of a situation which persists at higher orders, and which is treated in Sect. 6.

One immediate consequence is the following:

Corollary 4.2. The normal bundle $\hat{N}=\hat{T} \mathscr{N} / T Q_{x}$ of a sky $Q_{x}$ relative to $\mathscr{N}^{(1)}$ is isomorphic to

$$
\mathcal{O}(1,0) \oplus \mathcal{O}(1,0) \oplus \mathcal{O}(0,1) \oplus \mathcal{O}(0,1),
$$

where $\mathcal{O}(1,0)$ and $\mathcal{O}(0,1)$ denote the respective divisors of the two factors of $Q_{x} \cong \mathbb{P}_{1} \times \mathbb{P}_{1}$.

Proof. Indeed, we get something a bit more natural looking, namely a canonical isomorphism

$$
\hat{T} \mathscr{N} / T Q_{x} \cong\left[\left(\mathbf{S}_{+}\right)_{x} \otimes L_{-}\right] \oplus\left[\left(\mathbf{S}_{-}\right)_{x} \otimes L_{+}\right]
$$


where $L_{ \pm}$and $\hat{T} \mathscr{N}$ are to be interpreted as the restrictions of these to bundles to $Q_{x}$. For the tangent space of $Q_{x}$ at $\gamma$ corresponds to the set of Jacobi fields which vanish at $x \in \gamma$. Thus evaluating a solution of the generalized Jacobi equation at $x$ corresponds to projecting the corresponding element of $\widehat{T} \mathscr{N}$ to the normal bundle of $Q_{x}$. Since $\omega^{A}$ and $\xi^{A^{\prime}}$ have, respectively, homogeneities $(0,1)$ and $(1,0)$ in $\lambda$ and $\pi$, the result follows. Q.E.D.

This corollary, as we shall see in the next section, is a key ingredient in the proof of the main theorem.

We now examine the following question: What would have happened had we replaced the line bundle $L_{+} \rightarrow \mathscr{N}$ with a holomorphic line bundle $\tilde{L}_{+}$of the same Chern class? Writing $\tilde{L}_{+}=L_{+} \otimes E$, where $c_{1}(E)=0$, we can produce via the Ward correspondence a line bundle $\hat{E}$ on $\mathscr{M}$ with holomorphic connection $\nabla$, and produce two ersatz spinor bundles

$$
\widetilde{\mathbf{S}}_{+}=\mathbf{S}_{+} \otimes \hat{E}, \quad \tilde{\mathbf{S}}_{-}=\mathbf{S}_{-} \otimes \hat{E}^{-1}
$$

on $\mathscr{M}$, so that $T \mathscr{M}=\widetilde{\mathbf{S}}_{+} \otimes \widetilde{\mathbf{S}}_{-}$and such that the obvious connections on $\widetilde{\mathbf{S}}_{ \pm}$ induce the Levi-Civita connection on $T \mathscr{M}$ for any given choice of conformal scale. All of the above arguments then go through provided that all spinors are replaced by sections of $\widetilde{\mathbf{S}}_{ \pm}$and all covariant derivatives of spinors are "charged with respect to the electromagnetic field $(\hat{\mathrm{E}}, \nabla)$ " - i.e. interpreted in terms of the natural connections on $\widetilde{\mathbf{S}}_{ \pm}$. The ambidexterity again appears as the fact that $L_{+} \otimes E$ and $L_{-} \otimes E^{-1}$ give rise to the same thickening, albeit with different Poisson structures.

Let us now give a concrete description of the structure sheaf $\mathcal{O}_{(1)}$ of a first-order Poisson thickening determined by a line bundle $E \rightarrow X$. For any thickening of a complex manifold $X$, the extended tangent bundle $\hat{T} X$ and the inclusion $j: T X \rightarrow \hat{T} X$ determine the corresponding thickening by

$$
\mathcal{O}_{(1)}:=\left\{(f, \psi) \in \mathcal{O} \oplus \mathcal{O}\left[(\hat{T} X)^{*}\right] \mid j^{*} \psi=d f\right\},
$$

where $\left(f_{1}, \psi_{1}\right)\left(f_{2}, \psi_{2}\right):=\left(f_{1} f_{2}, f_{1} \psi_{2}+f_{2} \psi_{1}\right)$.

If we have a sub-bundle $\hat{D} \subset \hat{T} X$ with $\hat{T} X=\hat{D}+T X$, then, setting $D=\hat{D} \cap T X$, we have

$$
\mathcal{O}_{(1)}=\left\{(f, \varphi) \in \mathcal{O} \oplus \mathcal{O}\left(D^{*}\right)\left|j^{*} \varphi=d f\right|_{D}\right\},
$$

with $\left(f_{1}, \varphi_{1}\right)\left(f_{2}, \varphi_{2}\right)=\left(f_{1} f_{2}, f_{1} \varphi_{2}+f_{2} \varphi_{1}\right)$. For a Poisson thickening of a contact manifold $(X, D)$ determined by a line bundle $E \rightarrow X$, we have $\hat{D}=\left(L \otimes E^{*} \otimes J^{1} E\right) / \mathcal{O}$, where inclusion $j: D \rightarrow \hat{D}$ is induced by contraction with $d \theta$, and where the relevant trivial line sub-bundle of $L \otimes E^{*} \otimes J^{1} E$ is spanned by $\theta$. Now $\mathcal{O}\left(E^{*} \otimes J^{1} E\right)$ is just given by the 1 -forms on the total space of $E^{*}-0_{E^{*}}=E-0_{E}$ which are invariant under the action of $\mathbb{C}-\{0\}$, so that $\mathcal{O}\left(\hat{D}^{*}\right)$ is the space of vector fields on $E_{*}:=E-0_{E}$ with values in $L$ which are invariant under $\mathbb{C}-\{0\}$ and project to $D \subset T X:$

$$
\left.\pi^{-1} \mathcal{O}\left(D^{*}\right)=\left\{v \in \mathcal{O}_{E_{*}}\left(\pi^{*} L \otimes T E_{*}\right) \mid[\xi, v]=v\right\lrcorner \pi^{*} \theta=0\right\},
$$

where $\xi$ is the Euler vector field which generates the $(\mathbb{C}-\{0\})$ - action and where $\pi: E_{*} \rightarrow X$ is the canonical projection. Thus, thought of in terms of $E_{*}$,

$$
\left.\left.\pi^{-1} \mathcal{O}_{(1)}=\left\{(f, v) \in \mathcal{O} \oplus \mathcal{O}\left(\pi^{*} L \otimes T E_{*}\right) \mid \xi f=[\xi, v]=v\right\lrcorner \pi^{*} \theta=0,\left.d f\right|_{\pi_{*}^{-1} D}=v\right\lrcorner d \pi^{*} \theta\right\},
$$


and, again,

$$
\left(f_{1}, v_{1}\right)\left(f_{2}, v_{2}\right)=\left(f_{1} f_{2}, f_{1} v_{2}+f_{2} v_{1}\right)
$$

Now, on a Poisson-thickened contact manifold, we have noted the existence of a "divisor" line-bundle related to the Poisson transformations. In our present context, we can describe this very concretely, as follows. On $E_{*}$, consider the sheaf of vector fields $w$ which are $(\mathbb{C}-\{0\})$-invariant and project to contact vector fields, and let $\hat{\mathscr{C}}$ denote its direct image on $X$ :

$$
\pi^{-1} \hat{\mathscr{C}}:=\left\{w \in \mathcal{O}\left(T E_{*}\right) \mid \mathscr{L}_{w} \pi^{*} \theta \propto \pi^{*} \theta,[\xi, w]=0\right\} .
$$

This is then given an $\mathcal{O}_{(1)}$-module structure by setting

$$
(f, v) w=f w-\left\langle\pi^{*} \theta, w\right\rangle v
$$

which makes sense since, given that $\xi f=[\xi, v]=v\lrcorner \pi^{*} \theta=0$ and $\left.\left.d f\right|_{D}=v\right\lrcorner \pi^{*} \theta$, the assumptions that $[\xi, w]=0$ and $\mathscr{L}_{w} \pi^{*} \theta \equiv 0\left(\bmod \pi^{*} \theta\right)$ imply that

$$
\left.[\xi,(f, v) w]=\left[\xi, f w-(w\lrcorner \pi^{*} \theta\right) v\right]=0
$$

and

$$
\begin{aligned}
\mathscr{L}_{(f, v) w} \pi^{*} \theta & \left.=[(f, v) w]\lrcorner \pi^{*} d \theta+d[[(f, v) w]\lrcorner \pi^{*} \theta\right] \\
& \left.\left.=\left(f w-\left\langle\pi^{*} \theta, w\right\rangle v\right)\right\lrcorner \pi^{*} d \theta+d\left[\left(f w-\left\langle\pi^{*} \theta, w\right\rangle v\right)\right\lrcorner \pi^{*} \theta\right] \\
& \left.\equiv f w\lrcorner \pi^{*} d \theta-\left\langle\pi^{*} \theta, w\right\rangle d f+d(f w\lrcorner \pi^{*} \theta\right) \quad\left(\bmod \pi^{*} \theta\right) \\
& \left.=f w\lrcorner \pi^{*} d \theta+f d(w\lrcorner \pi^{*} \theta\right) \\
& =f \mathscr{L}_{w} \pi^{*} \theta \\
& \equiv 0 \quad\left(\bmod \pi^{*} \theta\right) .
\end{aligned}
$$

Moreover, the exact sequence

$$
0 \rightarrow \mathcal{O} \rightarrow \hat{\mathscr{C}} \rightarrow \mathcal{O}(L) \rightarrow 0,
$$

where the $\mathcal{O}$ factor consists of the multiples of $\xi$ and where the projection to $\mathcal{O}(L)$ is $w \mapsto \theta\left(\pi_{*} w\right)$, corresponds exactly to the restriction sequence relating an $\left.\mathcal{O}_{(1)}\right)^{-}$ module to two $\mathcal{O}$-modules; this shows that $\hat{\mathscr{C}}$ is locally free and of rank one over $\mathcal{O}_{(1)}$. Thus we may write

$$
\hat{\mathscr{C}}=\mathcal{O}_{(1)}(L)
$$

In fact, $\hat{\mathscr{C}}$ is actually the promised divisor line bundle related to infinitesimal $\tau$-preserving transformations. Indeed, every local section of $\hat{\mathscr{C}}$ defines a derivation of $\mathcal{O}_{(1)}$ by

$$
w \cdot(f, v):=(w f,[w, v]),
$$

where the Lie bracket $[w, v]$ is to be calculated relative to a local trivialization of $\pi^{*} L$ for which $\mathscr{L}_{w} \pi^{*} \vartheta=0$. This defines a map $\mathcal{O}_{(1)} \rightarrow \mathcal{O}_{(1)}$ because

$$
\left.v\lrcorner \pi^{*} \theta=0, \quad \mathscr{L}_{w} \pi^{*} \theta \propto \pi^{*} \theta \Rightarrow[w, v]\right\lrcorner \pi^{*} \theta=0,
$$


and

$$
\begin{aligned}
d(w f) & \left.=\mathscr{L}_{w} d f \equiv \mathscr{L}_{w}(v\lrcorner d \pi^{*} \theta\right) \quad\left(\bmod \pi^{*} \theta\right) \\
& =[w, v]\lrcorner d \pi^{*} \theta,
\end{aligned}
$$

where the last equality is most easily seen by taking a trivialization of $\pi^{*} L$ so that $\mathscr{L}_{w} d \pi^{*} \vartheta=0$, and hence so that $\mathscr{L}_{w} d \pi^{*} \vartheta=d_{w} \pi^{*} \vartheta=0$. This is actually a derivation of $\mathcal{O}_{(1)}$ because

$$
\begin{aligned}
w \cdot\left[\left(f_{1}, v_{1}\right)\left(f_{2}, v_{2}\right)\right] & =w \cdot\left(f_{1} f_{2}, f_{1} v_{2}+f_{2} v_{1}\right)=\left(w\left(f_{1} f_{2}\right),\left[w, f_{1} v_{2}+f_{2} v_{1}\right]\right) \\
& =\left(f_{1} w f_{2}+f_{2} w f_{1},\left(w f_{1}\right) v_{2}+\left(w f_{2}\right) v_{1}+f_{1}\left[w, v_{2}\right]+f_{2}\left(w, f_{1}\right]\right) \\
& \left.=\left(f_{1}, v_{1}\right)\left(w f_{2},\left[w, v_{2}\right]\right)+\left(f_{2}, v_{2}\right)\left(w f_{1}, w, v_{1}\right]\right) \\
& =\left(f_{1}, v_{1}\right) w \cdot\left(f_{2}, v_{2}\right)+\left(f_{2}, v_{2}\right) w \cdot\left(f_{1}, v_{1}\right) .
\end{aligned}
$$

The resulting homomorphism

$$
\mathcal{O}_{(1)}(L) \rightarrow \operatorname{Der}\left(\mathcal{O}_{(1)}\right)
$$

may be described locally by trivializing $E$ and $L$. Relative to local trivializations of $E$ and $L, \mathcal{O}_{(1)}$ becomes $\mathcal{O}[t] /\left(t^{2}\right)$, where $(f, v)$ corresponds to $\left[f+\frac{1}{s}\langle d s, v\rangle t\right]=$ $[f+\hat{f} t]$, where $s$ is the fiber coordinate on $E$ and where $v$ is thought of as a vector field on $E_{*}$ by employing the local trivialization of $L$. At the same time, $\hat{\mathscr{C}}=\mathcal{O}_{(1)}(L)$ is identified with $\mathcal{O}[t] /\left(t^{2}\right)$ by sending $w$ to $\left[\left\langle\vartheta, \pi_{*} w\right\rangle+\frac{1}{s}\langle d s, w\rangle t\right]=[g+\hat{g} t]$.
Now, choosing coordinates so that

$$
\vartheta=d u+\Sigma p_{j} d q^{j}
$$

a straightforward but tedious calculation shows that $w(f, v)$ corresponds to $\left\{f+t \hat{f}, \frac{1}{t}(g+t \hat{g})\right\}\left(\bmod t^{2}\right)$, where the Poisson bracket is taken with respect to the exelissic form

$$
\tau=t\left[\left(t \frac{\partial}{\partial t}+\sum p_{j} \frac{\partial}{\partial p_{j}}\right) \wedge \frac{\partial}{\partial u}+\sum \frac{\partial}{\partial q^{j}} \wedge \frac{\partial}{\partial p_{j}}\right] .
$$

Now the formal tangent space of contact deformations of a contact manifold $(X, D)$ is $H^{1}(X, \mathcal{O}(L))$, since $\mathscr{O}(L)=\mathscr{C}$ represents the infinitesimal contact transformation of $X$. If instead of considering infinitesimal moduli of contact manifolds, we consider the infinitesimal moduli of pairs $((X, D), E \rightarrow X)$ consisting of a contact manifold and a line bundle over it, the formal tangent space is $H^{1}(X, \hat{\mathscr{C}})=$ $H^{1}\left(X, \mathcal{O}_{(1)}(L)\right)$, since $\hat{\mathscr{C}}$ is exactly the Lie algebra of the pseudo-group of automorphisms

$$
\begin{aligned}
& E \stackrel{\hat{\Phi}}{\rightarrow} E \\
& \downarrow \downarrow \\
& X \stackrel{\Phi}{\longrightarrow} X
\end{aligned}
$$

of $E \rightarrow X$ for which $\Phi$ is contact map and $\hat{\Phi}$ is a bundle morphism. But the formal 
tangent space to the moduli space of Poisson-thickened contact manifold to first order is $H^{1}\left(X, \mathcal{O}_{(1)}(L) / \mathbb{C}\right)$. Our process of assigning a first-order Poisson thickening to every line bundle $E \rightarrow X$ on a contact manifold then induces a linear map $H^{1}\left(X, \mathcal{O}_{(1)}(L)\right) \rightarrow H^{1}\left(X, \mathcal{O}_{(1)}(L) / \mathbb{C}\right)$ which, by the above picture, is just the natural map induced by

$$
0 \rightarrow \mathbb{C} \rightarrow \mathcal{O}_{(1)}(L) \rightarrow \mathcal{O}_{(1)}(L) / \mathbb{C} \rightarrow 0 .
$$

We thus obtain the following innocuous-looking conclusion, which will turn out to have important consequences in Sect. 8:

Proposition 4.2. Infinitesimal deformations of pairs $((X, D), E \rightarrow X)$ and infinitesimal deformations of $X^{(1)}$ through Poisson-thickened contact manifolds are related by the exact sequence

$$
\cdots \rightarrow H^{1}(X, \mathbb{C}) \rightarrow H^{1}(X, \hat{\mathscr{C}}) \rightarrow H^{1}\left(X, \mathcal{O}_{(1)}(L) / \mathbb{C}\right) \rightarrow H^{2}(X, \mathbb{C}) \rightarrow \cdots
$$

of vector spaces.

To conclude this section, let us see the transition between Poisson thickenings and ordinary thickenings more explicitly at first order. First order thickenings with normal bundle $L \rightarrow \mathcal{N}$ are parameterized by $H^{1}\left(\mathscr{N}, T \mathscr{N} \otimes L^{-1}\right)$. But the map $\tau_{*}: H^{1}(\mathscr{N}, \mathcal{O} / \mathbb{C}) \rightarrow H^{1}\left(\mathscr{N}, T \mathscr{N} \otimes L^{-1}\right)$ factorizes through $H^{1}\left(\mathscr{N}, D \otimes L^{-1}\right)$, as is made clear by our previous analysis. The short exact sequences

$$
0 \rightarrow D \otimes L^{-1} \rightarrow T \mathcal{N} \otimes L^{-1} \rightarrow \mathcal{O} \rightarrow 0
$$

gives rise to the long exact sequence

$$
0 \rightarrow \mathbb{C} \rightarrow H^{1}\left(\mathscr{N}, D \otimes L^{-1}\right) \rightarrow H^{1}\left(\mathscr{N}, T \mathscr{N} \otimes L^{-1}\right) \rightarrow \cdots
$$

and it is not difficult to see that the extension class of

$$
0 \rightarrow D \rightarrow T \mathscr{N} \rightarrow L \rightarrow 0
$$

is in the kernel of $H^{1}\left(D \otimes L^{-1}\right) \rightarrow H^{1}\left(T \mathcal{N} \otimes L^{-1}\right)$, and so spans the kernel. This accounts for the fact that $L_{+}$and $L_{+} \otimes L^{-1}$ give rise to the same thickening. Moreover, the map $H^{1}(\mathcal{N}, \mathcal{O} / \mathbb{C}) \rightarrow H^{1}\left(\otimes, D \otimes L^{-1}\right)$ is injective, for via the Penrose transform this map becomes

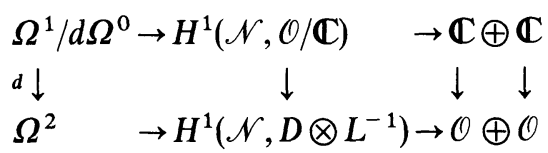

where we assume that $\mathscr{M}$ is, say, geodesically convex, hence Stein and contractible. Thus twisting by powers of $L$ gives rise to the only way of producing the same thickening from different line bundles.

\section{Extension to Third Order}

Given a Poisson thickening of $X$ of the first order, there is a direct manner in which one may consider the problem of extending it to third order. Indeed, let us consider 
the kernel of the restriction map $\mathscr{G}_{3} \rightarrow \mathscr{G}_{1}$; by construction, it is the exponential of $\mathscr{T}_{(2)}=\mathscr{T} / \mathscr{T}^{3}$ equipped with the Poisson bracket. However, $\{\mathscr{T}, \mathscr{T}\} \subset \mathscr{T}^{3}$, so we have an exact sequence

$$
\mathscr{T}_{(2)} \mapsto \mathscr{G}_{3} \rightarrow \mathscr{G}_{1}
$$

of sheaves of groups, where $\mathscr{T}_{(2)}$ is abelian and we can apply the adapted long exact sequence construction of $[E-L]$ or $[M]$. Namely, given $\mathbf{t} \in H^{1}\left(\mathscr{G}_{1}\right)$, the obstruction to $\mathrm{t}$ being in the image of $H^{1}\left(\mathscr{G}_{3}\right) \rightarrow H^{1}\left(\mathscr{G}_{1}\right)$ is an element of $H^{2}\left(\mathbf{t} \mathscr{T}_{(2)} \mathbf{t}^{-1}\right)$, and if this obstruction vanishes then $H^{1}\left(\mathbf{t} \mathscr{T}_{(2)} \mathbf{t}^{-1}\right)$ acts transitively on the inverse image of $t$ in $H^{1}\left(\mathscr{G}_{3}\right)$. If $X^{(1)}$ is the first order thickening of $X$ induced by $\mathbf{t}$, then $\mathbf{t} \mathscr{T}_{(2)} t^{-1}$ is a line bundle on $X^{(1)}$ whose restriction to $X$ is $\mathcal{O}\left(L^{*}\right)$; we simply need to understand the cohomology of such a creature.

Lemma 5.1. Let $\mathscr{N}^{(1)}$ denote the canonical first order thickening of an ambitwistor space, and let $\mathscr{L}_{(1)}^{*}$ denote any extension of $\mathcal{O}\left(L^{*}\right)$ to $\mathscr{N}^{(1)}$. Then $H^{1}\left(\mathscr{N}, \mathscr{L}_{(1)}^{*}\right)=$ $H^{2}\left(\mathscr{N}, \mathscr{L}^{*}{ }_{(1)}\right)=0$.

Proof. We have an exact sequence

$$
0 \rightarrow \mathcal{O}\left(L^{-2}\right) \rightarrow \mathscr{L}^{*} \rightarrow \mathcal{O}\left(L^{-1}\right) \rightarrow 0
$$

and since $H^{1}\left(\mathscr{N}, \mathcal{C}\left(L^{-2}\right)\right)=H^{2}\left(\mathscr{N}, \mathcal{C}\left(L^{-1}\right)\right)=0$, we are to verify that the Bockstein map

$$
\delta: H^{1}\left(\mathscr{N}, \mathcal{O}\left(L^{-1}\right)\right) \rightarrow H^{2}\left(\mathscr{N}, \mathcal{O}\left(L^{-2}\right)\right)
$$

is an isomorphism. As observed in Sect. 3, an element of $H^{1}\left(\mathscr{N}, \mathcal{O}\left(L^{-1}\right)\right)$ is determined by its restriction to first neighborhoods of skies $Q_{x} \subset \mathcal{N}$, and an element of $H^{2}\left(\mathcal{N}, \mathcal{O}\left(L^{-2}\right)\right)$ is determined by its restrictions to skies. It therefore follows that we need merely check that the Bockstein operator $\hat{\delta}: H^{1}\left(\mathscr{Q}_{x}, \mathcal{O}^{(1)}\left(L^{-1}\right)\right) \rightarrow H^{2}\left(Q_{x}, \mathcal{O}\left(L^{-2}\right)\right)$ of the short exact sequence $0 \rightarrow \mathcal{O}\left(L^{-2}\right) \rightarrow \hat{\mathcal{O}}^{(1)}\left(L^{-1}\right) \rightarrow \mathcal{O}^{(1)}\left(L^{-1}\right) \rightarrow 0$ is always an isomorphism, where $\mathcal{O}^{(1)}$ is the structure sheaf of the first neighborhood of $Q_{x} \subset \mathscr{N}^{(1)}$ and where $\hat{\mathcal{O}}^{(1)}$ is the structure sheaf of the first neighborhood of $Q_{x} \subset \mathscr{N}^{(1)}$.

Now let $N$ denote the normal bundle of $Q_{x} \subset \mathcal{N}$, and let $\hat{N}$ denote the normal bundle of $Q_{x} \subset \mathscr{N}^{(1)}$, we have the commutative diagram

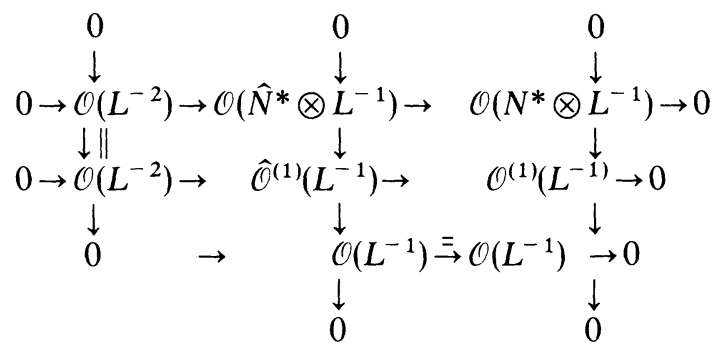

with exact rows and columns. Since $H^{*}\left(Q_{x}, \mathcal{O}\left(L^{-1}\right)\right)=H^{*}\left(\mathbb{P}_{1} \times \mathbb{P}_{1}, \mathcal{O}(-1,-1)\right)=0$, the Bockstein operator in question is precisely that of the top row:

$$
\hat{\delta}: H^{1}\left(Q_{x}, N^{*} \otimes L^{-1}\right) \rightarrow H^{2}\left(Q_{x}, L^{-2}\right) .
$$


But since $\hat{N} \cong 2 \mathcal{O}(1,0) \oplus 2 \mathcal{O}(0,1)$, as we saw in Sect. 3 .

$$
\begin{aligned}
H^{*}\left(Q_{x}, \mathcal{O}\left(\hat{N}^{*} \otimes L^{-1}\right)\right) & =H^{*}\left(\mathbb{P}_{1} \times \mathbb{P}_{1}, 2 \mathcal{O}(-2,-2) \oplus 2 \mathcal{O}(-1,-2)\right), \\
& =0
\end{aligned}
$$

and the Bockstein operator is therefore an isomorphism. Q.E.D.

This leads immediately to the following result:

Theorem 5.2. The canonical first order Poisson thickening of an ambitwistor space has a unique extension to a third order Poisson thickening.

\section{Ambidexterity of Obstructions}

Let $X$ be any complex contact manifold, let $\mathbf{t} \in H^{1}\left(X, \mathscr{G}_{m}\right), m \geqq 1$, be a Poisson thickening of $X$, let $\mathbf{r}=(\exp \tau)_{*}(\mathbf{t}) \in H^{1}\left(X, \operatorname{Aut}\left(\mathcal{O}_{m}\right)\right)$ be the corresponding equivalence class of "ordinary thickenings," and let $X^{(m)}$ be the thickening corresponding to $\mathbf{r}$. We may choose to forget that $X^{(m)}$ is a thickening of Poisson type, and simply ask whether or not $X^{(m)}$ can be extended as a thickening $X^{(m+1)}$. Alternatively, we may choose to be picky and ask whether $\mathbf{t}$ extends as a Poisson thickening. Surprisingly enough, these problems turn out to actually be equivalent.

Proposition 6.1. Let $\mathbf{u} \in H^{2}\left(X, \mathcal{O}\left(L^{-m}\right)\right)$ be the obstruction to extending to $H^{1}\left(X, \mathscr{G}_{m+1}\right)$, and let $\mathbf{v} \in H^{2}\left(X, \mathcal{O}\left(\hat{T} X \otimes L^{-m-1}\right)\right)$ be the obstruction to extending $\mathbf{r}=(\exp \tau)_{*}(\mathbf{t})$ to $H^{1}\left(X, \operatorname{Aut}\left(\mathcal{O}_{m+1}\right)\right)$. Then $\mathbf{u}=0 \Leftrightarrow \mathbf{v}=0$.

Proof. We have a commutative diagram

$$
\begin{array}{rlllll}
0 \rightarrow \quad \mathcal{O}\left(L^{-m}\right) & \rightarrow & \mathscr{G}_{m+1} & \rightarrow & \mathscr{G}_{m} & \rightarrow 1 \\
\downarrow & & \exp \downarrow & \rightarrow & \exp \downarrow
\end{array}
$$

with rows exact. If these sheaves of groups were abelian, we would have an induced commutative diagram relating the corresponding long exact sequences of cohomology groups. In the present non-abelian context, there is an analogous conclusion; namely, for any $\mathbf{t} \in H^{1}\left(X, \mathscr{G}_{m}\right)$, the obstructions $\mathbf{u}$ and $\mathbf{v}$ are related

$$
\mathbf{v}=\tau_{*}(\mathbf{u}) \text {, }
$$

where

$$
\tau_{*}: H^{2}\left(X, \mathcal{O}\left(L^{-m}\right)\right) \rightarrow H^{2}\left(X, \mathcal{O}\left(T X \otimes L^{-m-1}\right)\right)
$$

is induced by $\tau: \mathcal{O}\left(L^{-m}\right) \rightarrow \mathcal{O}\left([T X \oplus L] \otimes L^{-m-1}\right)$ via "twisting by t" - i.e. using $\left\{t_{\alpha \beta}\right\}$ as transition functions, where $\left\{t_{\alpha \beta}\right\}$ is a Čech representative for $\mathbf{t}$.

Now $\tau: \mathcal{O}\left(L^{-m}\right) \rightarrow \mathcal{O}\left([T X \oplus L] \otimes L^{-m-1}\right)$ has a canonical splitting $\sigma$ given by the contact form. Indeed, in terms of local coordinates $q^{0}, \ldots, q^{n}, p_{1}, \ldots, p_{n}, t$ we have

$$
\begin{aligned}
\tau\left(f t^{m}\right): & =\tau\lrcorner d\left(f t^{m}\right) \\
& =\left[\left(-m f+p_{j} f_{p_{j}}\right) \frac{\partial}{\partial q^{0}}+\left(f_{q} j-p_{j} f_{q^{0}}\right) \frac{\partial}{\partial p_{j}}-f_{p} \frac{\partial}{\partial q^{j}}\right] t^{m-1}+O\left(t^{m+2}\right)
\end{aligned}
$$


so that contraction with $\vartheta=d q^{0}+p_{j} d q^{j}$ yields

$$
\left.\tau\left(f t^{m}\right)\right\lrcorner \vartheta=-m f
$$

Hence $\sigma=\lrcorner\left(\frac{-1}{m} \theta\right)$ yields a splitting for $\left.\tau: \mathcal{O}\left(L^{-m}\right) \rightarrow \mathcal{O}(T X \oplus L] \otimes L^{-m-1}\right)$. The same will then also apply to the version twisted by $\mathbf{t}$; i.e.

$$
\tau: \mathcal{O}\left(L^{-m}\right) \rightarrow \mathcal{O}\left(\hat{T} X \otimes L^{-m-1}\right)
$$

is split by

$$
\sigma: \mathcal{O}\left(\widehat{T} X \otimes L^{-m-1}\right) \rightarrow \mathcal{O}\left(L^{-m}\right)
$$

defined as contraction with $\frac{-1}{m} \hat{\theta}: \hat{T} X \rightarrow L$.

But now

$$
\tau_{*}: H^{2}\left(X, \mathcal{O}\left(L^{-m}\right)\right) \rightarrow H^{2}\left(X, \mathcal{O}\left(\hat{T} X \otimes L^{-m-1}\right)\right)
$$

has left inverse given by $\sigma_{*}$. Thus $\mathbf{u}=\sigma_{*}(\mathbf{v})$ and $\mathbf{u}=0 \Leftrightarrow \mathbf{v}=0$.

This has two important consequences for our purposes. First off, it will allow us to "localize" our obstructions at skies. Secondly, it implies that the ambidexterity we noted for the first order thickening persists at higher orders, and that our results on the existence of higher order thickenings could be formulated without the present Poisson machinery, which may be viewed as a mere technical convenience.

Proposition 6.2. Suppose an ambitwistor space $\mathcal{N}$ has a thickening $\mathscr{N}^{(m)}, m \geqq 3$, extending the canonical first-order thickening $\mathscr{N}^{(1)}$. Then $\mathscr{N}^{(m)}$ is of Poisson type.

Proof. Let us begin by noticing that it suffices to prove the proposition for $m=3$. For the freedom of extension in passing from $\mathscr{N}^{(m-i)}$ to $\mathscr{N}^{(m)}$ is given $[E-L]$ by

$$
H^{1}\left(\mathscr{N}, \hat{T} \mathscr{N} \otimes L^{-m}\right)=\left\{\begin{array}{ll}
\mathcal{O}[-2] \oplus \mathcal{O}[-2], & m=2 \\
0, & m=3
\end{array},\right.
$$

where the expression on the right is calculated by Penrose transform in much the same manner explained in Sect. 3. Thus a thickening of Poisson type of order $m \geqq 3$ having an extension of Poisson type to order $m+1$ has only an extension of Poisson type. But Proposition 6.1 asserts that if there is an extension then there is one of Poisson type. This reduces the problem to showing that any third-order thickening extending the canonical first-order thickening must be of Poisson type.

We now consider the problem of extending the canonical first-order thickening directly to third order. There is a short exact sequence

$$
\operatorname{Der}_{\mathcal{O}_{(3)}}\left(\mathcal{O}_{(3)}, \mathscr{T}_{(3)^{\prime}}^{2}\right) \mapsto \operatorname{Aut}\left(\mathcal{O}_{(3)}\right) \rightarrow \operatorname{Aut}\left(\mathcal{O}_{(1)}\right),
$$

from whence we see that the freedom in extending $\mathscr{N}^{(1)}$ to $\mathscr{N}^{(3)}$ is given by $H^{1}\left(\mathcal{N}, \mathbf{t} \operatorname{Der}_{\mathcal{O}_{(3)}}\left(\mathcal{O}_{(3)}, \mathscr{T}_{(3)}^{2}\right) \mathbf{t}^{-1}\right)$, where $\mathbf{t} \in H^{1}\left(\mathscr{N}\right.$, Aut $\left.\mathcal{O}_{(1)}\right)$ is the isomorphism class of $\mathscr{N}^{(1)}$. But $\mathrm{t} \operatorname{Der}\left(\mathscr{C}_{(3)}, \mathscr{T}_{(3)}^{2}\right) \mathbf{t}^{-1}$ is a locally free sheaf of $\mathcal{O}_{(1)}$-modules whose restriction to $\mathscr{N}^{(0)}$ is $\mathcal{O}\left(\hat{T} \mathscr{N} \otimes L^{-2}\right)$; we therefore define

$$
\mathcal{O}_{(1)}\left(\hat{T} \mathscr{N} \otimes L^{-2}\right):=\mathbf{t} \operatorname{Der}\left(\mathcal{O}_{(3)}, \mathscr{T}_{(3)}^{2}\right) \mathbf{t}^{-1},
$$


so that we have an exact sequence

$$
0 \rightarrow \mathcal{O}\left(\hat{T} \mathscr{N} \otimes L^{-1}\right) \rightarrow \mathcal{O}_{(1)}\left(\widehat{T} \mathscr{N} \otimes L^{-2}\right) \rightarrow \mathcal{O}\left(\hat{T} \mathcal{N} \otimes L^{-2}\right) \rightarrow 0
$$

of $\mathcal{O}_{(1)}$-modules. Since $H^{1}\left(\mathscr{N}, \mathcal{O}\left(\hat{T} \mathscr{N} \otimes L^{-3}\right)\right)=0$, we have

$$
\begin{aligned}
& H^{1}\left(\mathscr{N}, \mathbf{t} \operatorname{Der}\left(\mathcal{O}_{(3)}, \mathscr{T}_{(3)}^{2}\right) \mathbf{t}^{-1}\right) \\
& \quad=\operatorname{Ker}\left[\delta: H^{1}\left(\mathscr{N}, \mathcal{O}\left(\hat{T} \mathscr{N} \otimes L^{-2}\right)\right) \rightarrow H^{2}\left(\mathscr{N}, \mathcal{O}\left(\hat{T} \mathscr{N} \otimes L^{-3}\right)\right)\right],
\end{aligned}
$$

where $\delta$ is the Bockstein map of the above short exact sequence. But here $H^{1}\left(\mathcal{N}, \mathcal{O}\left(\hat{T} \mathscr{N} \otimes L^{-2}\right)\right)$ exactly represents the freedom of extending $\mathscr{N}^{(1)}$ to $\mathscr{N}^{(2)}$, and this argument actually shows that the map from second-order freedom to third-order obstruction is an affine map.

Let us now analyze this map in light of what we already know about Poisson extensions. Corresponding to an original choice of $L_{ \pm}$in constructing our first order thickening, we have two canonical and linearly independent surjections $\theta_{ \pm}: \hat{T} \mathscr{N} \rightarrow L$ which extend the contact from $\theta: T \mathscr{N} \rightarrow L$. These induce maps

$$
\theta_{ \pm}: H^{1}\left(\mathcal{N}, \mathcal{O}\left(\hat{T} \mathcal{N} \otimes L^{-2}\right)\right) \rightarrow H^{1}\left(\mathcal{N}, \mathcal{O}\left(L^{-1}\right)\right)
$$

which exactly read off the two factors of

$$
H^{1}\left(\mathscr{N}, \mathcal{O}\left(T \mathscr{N} \otimes L^{-2}\right)\right)=\mathcal{O}[-2] \oplus \mathcal{O}[-2]
$$

They also induce maps

$$
\theta_{ \pm}: H^{2}\left(\mathscr{N}, \mathcal{O}\left(\widehat{T} \mathscr{N} \otimes L^{-3}\right)\right) \rightarrow H^{2}\left(\mathscr{N}, \mathcal{O}\left(L^{-2}\right)\right)
$$

which correspond to reading off the obstruction to Poisson extension, à la Proposition 6.1, relative to the two different Poisson structures. The linear map

$$
\begin{gathered}
\theta_{+} \delta \oplus \theta_{-} \delta: H^{1}\left(\mathcal{N}, \mathcal{O}\left(\widehat{T} \mathscr{N} \otimes L^{-2}\right)\right) \rightarrow\left[H^{2}\left(\mathcal{N}, \mathcal{O}\left(L^{-2}\right)\right)\right]^{\oplus 2} \\
: \mathcal{O}[-2] \oplus \mathcal{O}[-2] \rightarrow \mathcal{O}[-2] \oplus \mathcal{O}[-2]
\end{gathered}
$$

is therefore precisely the direct sum of the two Bockstein operators measuring the obstruction to extending a second order Poisson thickening to third order relative to the two available Poisson structures. Since we saw in Sect. 5 that each of the latter operators is an isomorphism, it follows that $\operatorname{ker} \delta=0$, and there is at most one third-order thickening $\mathscr{N}^{(3)}$. Since we proved in Sect. 5 that there is, in fact, a third-order Poisson thickening, it follows that the unique $\mathscr{N}^{(3)}$ exists and is of Poisson type. Q.E.D.

Corollary 6.3. ("Ambidexterity Theorem"). For any ambitwistor space $\mathcal{N}$, the "right handed" Poisson thickenings of order $m$, obtained by extending the canonical first order thickening constructed from $L_{+}$, and the "left handed" Poisson thickenings of order in $m$, obtained by instead beginning with $L_{-}$, define exactly the same ringed spaces $\mathcal{N}^{(m)}, m \geqq 3$. (Of course these spaces are thereby equipped with different auxiliary structures.)

Corollary 6.4. ("Equivalence Theorem"). The canonical first-order thickening $\mathscr{N}^{(1)}$ of an ambitwistor space has an extension to an $m^{\text {th }}$ order thickening $\mathscr{N}^{(m)}, m \geqq 2$, iff it has an $m^{\text {th }}$ order Poisson extension. 


\section{Legendrian and Poisson Fattenings}

Up until the present we have imagined the Poisson thickenings of complex contact manifolds as arising in two stages: first one constructs or is given a complex contact manifold $(X, D)$, and then one considers its Poisson thickenings as defined by $H^{1}\left(X, \mathscr{G}_{m}\right)$. However, one may instead construct Poisson thickenings in one step by assembling $X^{(m)}$ from copies of the $m^{\text {th }}$ infinitesimal neighborhood of $\mathbb{C}^{2 n+1}$ via the action of a certain pseudo-group of transformations. form

Let $\mathbb{C}^{2 n+1}$ be given standard coordinates $u, q^{1}, \ldots, q^{n}, p_{1}, \ldots, p_{n}$ and the contact

$$
\vartheta=d u+\sum_{j=1}^{n} p_{j} d q^{j}
$$

Let $\mathscr{C}$ denote the pseudo-group of contact transformations of $\mathbb{C}^{2 n+1}$ :

$$
\mathscr{C}=\left\{\varphi: U \rightarrow V \text { biholomorphic } \mid U, V \subset \mathbb{C}^{2 n+1},{ }_{\exists} f: U \rightarrow \mathbb{C}-\{0\}, \varphi^{*} \vartheta=f \vartheta\right\} .
$$

Then $\mathscr{C}$ acts naturally on $\mathbb{C}^{2 n+2}:$ for all $\varphi: U \rightarrow V$ in $\mathscr{C}$ let

$$
\tilde{\varphi}: U \times \mathbb{C} \rightarrow V \times \mathbb{C}
$$

be defined by $\tilde{\varphi}(z, t): f=(\varphi(z), f(z) t)$, where $\varphi^{*} \vartheta=f \vartheta$. Thus $\mathbb{C}^{2 n+2}$, which we equip with coordinates $u, q^{1}, \ldots, q^{n}, p_{1}, \ldots, p_{n}, t$, is just being thought of as the contact line bundle $L$ of our contact structure on $\mathbb{C}^{2 n+1}$, and $\mathscr{C}$ acts on $L=\mathbb{C}^{2 n+2}$ in the natural fashion. Since this action preserves the "zero section" $\mathbb{C}^{2 n+1} \subset \mathbb{C}^{2 n+2}$, we also get an action of $\mathscr{C}$ on all of the infinitesimal neighborhoods $\left(\mathbb{C}^{2 n+1}\right)^{(m)}$, of $\mathbb{C}^{2 n+1} \subset \mathbb{C}^{2 n+2}$.

Now the sheaf $\mathscr{G}_{m}$ on $\mathbb{C}^{2 n+1}$ acts on $\left(\mathbb{C}^{2 n+1}\right)^{(m)}$, and the action of $\mathscr{C}$ sends $\mathscr{G}_{m}$ to itself. We may therefore define a new pseudo-group

$$
\mathscr{C}_{m}=\mathscr{C} \bowtie \mathscr{G}_{m}
$$

as the semi-direct product on $\mathscr{C}$ and $\mathscr{G}_{m}$; here $\mathscr{C}$ acts on $\mathscr{G}_{m}$ by conjugation. Thus $\mathscr{C}_{m}$ is the pseudo-group of transformations of $\left(\mathbb{C}^{2 n+1}\right)^{(m)}$ generated by the contact transformations and our Poisson automorphisms. It follows that Poisson-thickened contact manifolds are precisely those objects constructed by gluing together pieces of $\left(\mathbb{C}^{2 n+1}\right)^{(m)}$ with the transformations $\mathscr{C}_{m}$.

The Lie algebra of $\mathscr{C}_{m}$ is a sheaf on $\mathbb{C}^{2 n+1}$ of vector fields on $\left(\mathbb{C}^{2 n+1}\right)^{(m)}$. Since the infinitesimal contact transformations of $\mathbb{C}^{2 n+1}$ are exactly given by expressions of the form $\tau\lrcorner d(f(z) / t)$, it is easy to see that this new Lie algebra is precisely given by vector fields of the $\tau\lrcorner d(g / t)$, where $g \in \mathcal{O}_{(m+1)}$. Thus, if $\mathscr{L}_{(m)}$ denotes the restriction to $\left(\mathbb{C}^{2 n+1}\right)^{(m)}$ of the divisor line bundle of $\mathbb{C}^{2 n+1} \subset \mathbb{C}^{2 n+2}$, the Lie algebra of $\mathscr{C}_{m}$ is precisely $\mathbb{C}_{m}:=\left(\mathscr{L}_{(m)} / \mathbb{C},\{\},\right)$. We thus have an exact sequence

$$
0 \rightarrow \mathscr{A}_{m} \rightarrow \mathfrak{C}_{m} \rightarrow \mathfrak{C} \rightarrow 0,
$$

where $\mathbb{C}$ is the Lie algebra of contact vector fields on $\mathbb{C}^{2 n+1}$.

We now wish to consider the entire construction problem relative to a Legendrian submanifold $\Sigma \subset X$; i.e. relative to $\Sigma^{n} \subset X^{2 n+1}$ such that $T \Sigma \subset D$. To do this we will first need to understand the normal bundle of such a creature.

Lemma 7.1. Let $(X, D)$ be a complex contact manifold, let $\Sigma \subset X$ be a Legendrian submanifold, and let $L_{\Sigma}$ denote the restriction of $\Sigma$ of the contact line bundle 
$L=T X / D$. Then the normal bundle $N=\left(\left.T X\right|_{\Sigma}\right) / T \Sigma$ is given by

$$
N=J^{1} L_{\Sigma}
$$

Proof. For any complex contact manifold we recall that $L^{*}-0_{L^{*}}$ is a symplectic manifold, while the inverse image of a Legendrian submanifold $\Sigma \subset X$ is a Lagrangian submanifold $\pi^{-1}(\Sigma) \subset L^{*}-0_{L^{*}}$. Since $\pi^{-1}(\Sigma)$ is Lagrangian, its normal bundle is isomorphic to its cotangent bundle. The result then follows from the fact that $J^{1} L=T^{*} L^{*} / \mathbb{C}_{*}$ by restricting this isomorphism to $\pi^{-1}(\Sigma)$ to obtain

$$
N=T^{*}\left(\pi^{-1}(\Sigma)\right) / \mathbb{C}_{*}=J^{1} L_{\Sigma}
$$

Now for any manifold $\Sigma$ and any line bundle $L_{\Sigma} \rightarrow \Sigma$, the total space of $J^{1} L_{\Sigma}$ carries a natural contact structure. ${ }^{1}$ Indeed, if $\mathfrak{P}: J^{1} L_{\Sigma} \rightarrow \Sigma$ is the canonical projection, then both $\mathfrak{P}^{*} L_{\Sigma}$ and $\mathfrak{P}^{*} J^{1} L_{\Sigma}$ have canonical sections, the former being obtained from the latter via the projection $J^{1} L_{\Sigma} \rightarrow L_{\Sigma}$. Let us call these sections $\sigma_{1} \in \Gamma\left(J^{1} L_{\Sigma}, \mathfrak{P}^{*} L_{\Sigma}\right)$ and $\sigma_{2} \in \Gamma\left(J^{1} L_{\Sigma}, \mathfrak{B}^{*} J^{1} L_{\Sigma}\right)$. The natural inclusion $l: \mathfrak{P}^{*} J^{1} L_{\Sigma} \hookrightarrow$ $J^{1} \mathfrak{P}^{*} L_{\Sigma}$ then may be used to compare our two sections and we may set $\theta=J^{1}\left(\sigma_{1}\right)-\imath \sigma_{2}$. By construction, $\theta$ is in the kernel of $J^{1} \mathfrak{P}^{*} L_{\Sigma} \rightarrow \mathfrak{P}^{*} L_{\Sigma}$, and thus is a section of $\Omega^{1}\left(\mathfrak{P}^{*} L_{\Sigma}\right)$ on the total space of $J^{1} L_{\Sigma}$.

To check that $\theta$ is a contact form, we take a local trivialization of $L_{\Sigma}$, identifying $L_{\Sigma}$ with the trivial line bundle $\mathcal{O}$, and thereby identifying $J^{1} L_{\Sigma}$ with $\mathcal{O} \oplus T^{*} \Sigma$; this also trivializes $\mathfrak{P}^{*} L_{\Sigma}$, and $J^{1} \mathfrak{P}^{*} L_{\Sigma}$ becomes $\mathcal{O} \oplus T^{*}\left(J^{1} L_{\Sigma}\right)$. Choosing coordinates $q^{1}, q^{n}$ on $\Sigma$, we have coordinates $u, q^{1}, \ldots q^{n}, p_{1}, \ldots, p_{n}$ on $J^{1} L_{\Sigma}$, so that

$$
\begin{aligned}
\sigma_{1} & =u, \\
J^{1}\left(\sigma_{1}\right) & =u \oplus d u . \\
\sigma_{2} & =u \oplus\left(-\Sigma p_{j} d q^{j}\right), \\
l \sigma_{2} & =u \oplus\left(-\Sigma p_{j} d q^{j}\right),
\end{aligned}
$$

and $\theta=J^{1}\left(\sigma_{1}\right)-\imath \sigma_{2}=0 \oplus\left(d u+\Sigma p_{j} d q^{j}\right)$. This shows that $\theta$ is indeed a contact form.

Now given a Legendrian submanifold $\Sigma \subset X$, we may ask how $X$ compares to our standard contact manifold $J^{1} L_{\Sigma}$. Certainly we may find local isomorphisms which induce the identity on the normal bundle level. If $\mathscr{C}_{\Sigma}$ is the sheaf on $\Sigma$ of germs at $\Sigma$ of contact automorphisms of $J^{1} L_{\Sigma}$ acting trivially on the zero section $\Sigma$ and on its normal bundle, the germ of $\Sigma \subset X$ is therefore given up to contact isomorphism by an element of $H^{1}\left(\Sigma, \mathscr{C}_{\Sigma}\right)$.

Let $\mathscr{J} \subset \mathcal{O}$ denote the ideal of the zero section in the sheaf of germs of holomorphic functions on $J^{1} L_{\Sigma}$, restricted to the zero section. For each $\varphi \in \mathscr{C}_{\Sigma}$, we have $\varphi^{*}(\mathscr{J})=\mathscr{J}$, and hence $\varphi^{*}\left(\mathscr{J}^{\ell}\right)=\mathscr{J}^{\ell}$ for all $\ell$. Let $\mathscr{S}_{\ell}$ denote the subgroup of $\mathscr{C}_{\Sigma}$ consisting of transformations $\varphi$ satisfying

(1) $\varphi^{*}$ induces the identity on $\mathcal{O} / \mathscr{J}^{\ell+1}$, and

(2) $\varphi^{*}$ induces the identity on $\mathscr{J} / \mathscr{J}^{\ell+2}$.

Clearly this is a normal subgroup, and we will define

$$
\mathscr{B}_{\ell}=\mathscr{C}_{\Sigma} / \mathscr{S}_{\ell}
$$

\footnotetext{
${ }^{1}$ Note added in proof: This of course reflects the fact that $J^{\prime} L_{\Sigma}$ is the open subset of $\mathbb{P}\left(T^{*} L_{\Sigma}\right)$ consisting of hyperplanes transverse to the fibers.
} 
Elements of $H^{1}\left(\Sigma, \mathscr{B}_{\ell}\right)$ will be called Legendrian fattenings of $\Sigma$ of order $\ell$; a Legendrian imbedding $\Sigma \hookrightarrow X$ determines an entire hierarchy of such objects, which are roughly its $\ell$-jets. Since $\mathscr{B}_{\ell} \subset \operatorname{Aut}\left(\mathcal{O} / \mathscr{J}^{\ell+1}\right)$, a Legendrian fattening determines a fattening in the sense discussed in [E - L2]. But a Legendrian fattening also comes equipped with an "extended ideal sheaf" corresponding to $\mathscr{J} / \mathscr{J}^{\ell+2}$ which ostensibly contains information about the ordinary fattening of order $l+1$.

The reason for considering these transformations and the corresponding "fattenings" becomes clear when one considers the associated Lie algebras. Relative to the contact form $\vartheta=d u+\Sigma p_{j} d q^{j}$, consider the infinitesimal contact transformation induced by a section of $\mathfrak{P}^{*} L_{\Sigma}$ represented by a holomorphic function $f$; it is given by

$$
\xi_{f}=\left(-f+\Sigma p_{j} f_{p_{j}}\right) \frac{\partial}{\partial u}-\Sigma f_{p_{j}} \frac{\partial}{\partial q^{j}}+\Sigma\left(f_{q} j-p_{j} f_{u}\right) \frac{\partial}{\partial p_{j}}
$$

If $\Sigma$ is given by $p_{j}=u=0$, and if $\mathscr{J}=\left(p_{j}, u\right)$ is the ideal of $\Sigma$, we read off the fact that $\xi_{f}$ vanishes along $\Sigma$ iff $f \in \mathscr{J}^{2}\left(\mathfrak{P}^{*} L_{\Sigma}\right)$ and such that a $\xi_{f}$ must then induce the zero map on $\mathscr{J} / \mathscr{J}^{2}$. Thus $\mathscr{J}^{2}$ is the Lie algebra of $\mathscr{S}_{0}=\mathscr{C}_{\Sigma}$. More generally, $\xi_{f}$ induces the zero maps on $\mathcal{O} / \mathscr{J}^{\ell+1}$ and on $\mathscr{J} / \mathscr{J}^{\ell+2}$ iff $f \in \mathscr{J}^{\ell+2}\left(\mathfrak{P}^{*} L_{\Sigma}\right)$. Thus $\mathscr{J}^{\ell+2}\left(\mathfrak{P}^{*} L_{\Sigma}\right)$ is the Lie algebra of $\mathscr{S}_{\ell}$, and the fact that $\mathscr{S}_{\ell}$ is a normal subgroup corresponds to the fact that

$$
\left\{\mathscr{J}^{2}\left(\mathfrak{P}^{*} L_{\Sigma}\right), \mathscr{J}^{m+2}\left(\mathfrak{P}^{*} L_{\Sigma}\right)\right\} \subset \mathscr{J}^{m+2}\left(\mathfrak{B}^{*} L_{\Sigma}\right),
$$

so that $\mathscr{J}^{m+2}\left(\mathfrak{P}^{*} L_{\Sigma}\right)$ is an ideal in $\mathscr{J}^{2}\left(\mathfrak{P}^{*} L_{\Sigma}\right)$.

The obstruction theory for Legendrian fattenings now follows the pattern of our previous yoga; we have a short exact sequence

$$
\mathscr{J}^{\ell+2}\left(\mathfrak{P}^{*} L_{\Sigma}\right) / \mathscr{J}^{\ell+3}\left(\mathfrak{P}^{*} L_{\Sigma}\right) \longmapsto \mathscr{B}_{\ell+l} \rightarrow \mathscr{B}_{\ell},
$$

where

$$
\begin{aligned}
\mathscr{S}_{\ell} / \mathscr{S}_{\ell+1} & =\mathscr{J}^{\ell+2}\left(\mathfrak{P}^{*} L_{\Sigma}\right) / \mathscr{J}^{\ell+3}\left(\mathfrak{P}^{*} L_{\Sigma}\right) \\
& =\mathcal{O}\left(L_{\Sigma} \otimes \odot^{\ell+2} N^{*}\right),
\end{aligned}
$$

where $N=J^{1} L_{\Sigma}$ is our normal bundle. Since $\mathscr{S}_{\ell} / \mathscr{S}_{1+1}$ is in the center of $\mathscr{S}_{\ell+1}$, we obtain

Lemma 7.2. The obstruction to extending a Legendrian fattening of $\Sigma$ from order $\ell$ to order $\ell+1$ is an element of $H^{2}\left(\Sigma, O\left(L_{\Sigma} \otimes \odot^{\ell+2} N^{*}\right)\right)$. If this obstruction vanishes, the freedom of extension is given by an action of $H^{1}\left(\Sigma, \mathcal{O}\left(L_{\Sigma} \otimes \odot^{\ell+2} N^{*}\right)\right)$.

We now consider the analog of the above construction that arises when the contact transformations $\mathscr{C}$ are replaced with the Poisson transformations $\mathscr{C}_{m}$. We again begin with considerations pertaining to the normal bundle of $\Sigma$, this time relative to a Poisson-thickened contact manifold. Given such an imbedding $\Sigma \hookrightarrow X^{(m)}$, one may read off the normal bundle of $\Sigma$ from $L_{\Sigma}$ and the restriction $\alpha \in H^{1}(\Sigma, \mathcal{O} / \mathbb{C})$ of the class in $H^{1}\left(X, \mathscr{G}_{m}\right)$ determining the Poisson thickening; this follows from the fact that sections of $\mathcal{O}_{m} / \mathbb{C}$ which vanish along $\Sigma$ generate vector fields which vanish to second order along $\Sigma$. Let us now build our normal bundle $\hat{N}$ explicitly from $L_{\Sigma}$ and $\alpha$.

Let $L_{\Sigma} \rightarrow \Sigma$ and $\alpha \in H^{1}(\Sigma, \mathcal{O} / \mathbb{C})$ be given. Then $d \alpha \in H^{1}\left(\Sigma, \Omega^{1}\right)$ specifies an 
extension

$$
0 \rightarrow \Omega^{1}\left(L_{\Sigma}\right) \rightarrow V \rightarrow L_{\Sigma} \rightarrow 0 .
$$

We then obtain $\hat{N} \rightarrow \Sigma$ by pushing this extension out to $N=J^{1} L_{\Sigma}$; i.e. $\hat{N}=$ $\left(V \oplus J^{1} L_{\Sigma}\right) / \Omega^{1}\left(L_{\Sigma}\right)$, so that we have exact sequences

$$
0 \rightarrow \Omega^{1}\left(L_{\Sigma}\right) \rightarrow \hat{N} \rightarrow L_{\Sigma} \oplus L_{\Sigma} \rightarrow 0
$$

and

$$
0 \rightarrow J^{1} L_{\Sigma} \rightarrow \hat{N} \rightarrow L_{\Sigma} \rightarrow 0 .
$$

I claim that the total space of $N$ then carries a Poisson structure, and $N \subset \hat{N}$ gives rise to Poisson thickenings of all orders of the contact manifold $N=J^{1} L_{\Sigma}$ constructed above.

Letting $\mathfrak{P}: N \rightarrow \Sigma$ again denote the canonical projection, and letting $L=\mathfrak{P}^{*} L_{\Sigma}$ denote the contact line bundle of $N$, we let $\hat{\mathfrak{P}}: L \rightarrow \Sigma$ denote the composition of $\mathfrak{P}$ with the canonical projection $L \rightarrow N$. Then $\hat{\mathfrak{P}}^{*} \alpha \in H^{1}(L, \mathcal{O} / \mathbb{C})$ satisfies $0=\left\{\hat{\mathfrak{P}}^{*} \alpha, \hat{\mathfrak{P}}^{*} \alpha\right\} \in H^{2}(L, \mathcal{O})$ because, relative to the exelissic form

$$
\tau=t\left[\left(t \frac{\partial}{\partial t}+\Sigma p_{j} \frac{\partial}{\partial p_{j}}\right) \wedge \frac{\partial}{\partial u}+\Sigma \frac{\partial}{\partial q^{j}} \wedge \frac{\partial}{\partial p_{j}}\right] .
$$

two functions of $q$ have vanishing Poisson bracket. Thus $\exp \left(\hat{\mathfrak{P}}^{*} \alpha\right)$ is a well-defined element of $H^{1}\left(\mathscr{N}, \mathscr{G}_{\infty}\right)$, where $\mathscr{G}_{\infty}$ is the sheaf of germs along $N \subset L$ of Poisson transformations generated by $\mathcal{O} / \mathbb{C}$ using $\tau$. In fact, I claim that the image of $\exp \left(\hat{\mathfrak{P}}^{*} \alpha\right)$ in $H^{1}\left(N, \operatorname{Aut}\left(\mathcal{O}_{L}\right)\right)$ is simply the germ of the imbedding $N \hookrightarrow \hat{N}$ constructed above. Indeed, let us notice that, to a function $f(q), \tau$ associates the vector field

$$
V_{f}=t \Sigma f_{q^{j}} \frac{\partial}{\partial p_{j}} .
$$

(Note that this is the Poisson vector field associated to $f$, in contrast with $\xi_{f}$, which is associated to $f / t$.) Thus $V_{f}=t d f$, where the section $t d f$ of $\Omega^{1}\left(L_{\Sigma}\right)$ is reinterpreted as a vertical vector field on $J^{1} L_{\Sigma} \supset\left(T^{*} \Sigma\right) \otimes L_{\Sigma}$ which is linear up the fibers and vanishes along $N \subset L$. Identifying $L=\mathfrak{P}^{*} L_{\Sigma}$ with $L_{\Sigma}$ with $L_{\Sigma} \oplus J^{1} L_{\Sigma}$, this vector field corresponds to the endomorphism of $L \oplus J^{1} L_{\Sigma}$ with block-matrix form

$$
\left[\begin{array}{l|l}
0 & d f \\
\hline 0 & 0 \\
0 &
\end{array}\right]
$$

and in particular has square zero; thus $\exp (t d f)$ is just given by

$$
\left[\begin{array}{l|l}
1 & d f \\
\hline 0 & I \\
0 &
\end{array}\right]
$$

and $\exp \left(\hat{\mathfrak{P}}^{*} \alpha\right)$ consequently corresponds precisely to the push-out of $d \alpha$ to $J^{1} L_{\Sigma}$, as claimed. 
The upshot of all this is the following: to every pair $\left(L_{\Sigma}, \alpha\right)$ we may associated Poisson-thickened Legendrian embeddings of $\Sigma$ of all orders, and any Poissonthickened Legendrian embedding is obtained from one of these standard models by changing its transition functions by elements of $\mathscr{G}_{m}$ which preserves $\Sigma$ and act trivially on the normal bundle of $\Sigma$.

Thus, let $\Sigma \subset \hat{N}$ denote the zero section, let $\mathcal{O}_{(m)}$ denote the structure sheaf of the $m^{\text {th }}$ neighborhood of $N \subset \hat{N}$, let $\mathscr{K}_{m} \subset \mathcal{O}_{(m)}$ denote the ideal of functions vanishing along $\Sigma$, and let $\mathscr{G}_{m \Sigma}$ denote the sheaf of germs at $\Sigma$ of Poisson transformations (i.e. elements of $\mathscr{G}_{m}$ ) which fix $\Sigma$ and act trivially on $\mathscr{K}_{m} / \mathscr{K}_{m}^{2}$. Then, for some $\left(L_{\Sigma}, \alpha\right)$, the germ of any Poisson-thickened Legendrian imbedding of $\Sigma$ is exactly given by some element of $H^{1}\left(\Sigma, \mathscr{G}_{m \Sigma}\right)$.

We now filter $\mathscr{G}_{m \Sigma}$ in analogy to our previous work. Let $\mathscr{S}_{m, r}$ denote the normal subgroup of $C_{m \Sigma}$ consisting of transformations $\varphi$ such that

(1) $\varphi^{*}$ induces the identity on $\mathcal{O} / \mathscr{K}_{m}^{\ell+1}$;

and (2) $\varphi^{*}$ induces the identity on $\mathscr{K}_{m} / \mathscr{K}_{m}^{\ell+2}$.

Set

$$
\mathscr{B}_{m, \ell}:=\mathscr{C}_{m \Sigma} / \mathscr{S}_{m, \ell}
$$

We will then call elements of $H^{1}\left(\Sigma, \mathscr{B}_{m, \ell}\right)$ Poisson fattenings of $\Sigma$ of type $(m, \ell)$. We have a family of central extensions

where

$$
\mathscr{K}_{m}^{\ell+2}\left(\hat{\mathfrak{P}}^{*} L_{\Sigma}\right) / \mathscr{K}_{m}^{\ell+3}\left(\hat{\mathfrak{P}}^{*} L_{\Sigma}\right) \nrightarrow \mathscr{B}_{m, \ell+1} \rightarrow \mathscr{B}_{m, \ell}
$$

$$
\begin{aligned}
\mathscr{S}_{m, \ell} / \mathscr{S}_{m, \ell+1} & =\mathscr{K}_{m}^{\ell+2}\left(\hat{\mathfrak{P}}^{*} L_{\Sigma}\right) / \mathscr{K}_{m}^{\ell+3}\left(\hat{\mathfrak{P}}^{*} L_{\Sigma}\right) \\
& =\left\{\begin{array}{l}
\mathcal{O}\left(L_{\Sigma} \otimes \odot_{m}^{\ell+2} \hat{N}^{*}\right), \quad \ell<m-2 \\
\mathcal{O}\left(L_{\Sigma} \otimes\left[\odot^{\ell+2} \hat{N}^{*} / L_{\Sigma}^{-(m+1)} \otimes \odot^{\ell-m+1} \hat{N}^{*}\right]\right), \quad \text { otherwise }
\end{array}\right.
\end{aligned}
$$

since the Lie algebra of $\mathscr{S}_{m, \ell}$ is precisely $\mathscr{K}_{m}^{\ell+2}\left(\hat{\mathfrak{P}}^{*}\right)$ via the same sort of Poisson bracket calculation as before. This yields

Lemma 7.3. For $\ell \geqq 1$, the obstruction to extending a Poisson fattening of type $(m, \ell)$ to one of type $(m, \ell+1)$ is an element of $H^{2}\left(\Sigma, \mathcal{O}\left(L_{\Sigma} \otimes \odot^{\ell+2} \hat{N}^{*}\right)\right)$ if $m>\ell+2$, and is otherwise an element of $H^{2}\left(\Sigma, \mathcal{O}\left(L_{\Sigma} \otimes\left[\odot^{\ell+2} \hat{N}^{*} / L_{\Sigma}^{-(m+1)} \otimes \odot^{\ell-m+1} \hat{N}^{*}\right]\right)\right)$. The freedom of such extension is parameterized by $H^{1}\left(\Sigma, \mathcal{O}\left(L_{\Sigma} \otimes \odot^{\ell+2} \hat{N}^{*}\right)\right)$ if $m>\ell+2$, and is otherwise an element of $H^{1}\left(\Sigma, \mathcal{O}\left(L_{\Sigma} \otimes\left[\odot^{\ell+2} \hat{N}^{*} / L_{\Sigma}^{-(m+1)} \otimes \odot^{\ell-m+1} \hat{N}^{*}\right]\right)\right)$.

There is one more obstruction which will be of great importance in the rest of this paper; namely, given a Poisson fattening of type $(m, \ell)$, when can one extend it to type $(m+1, \ell)$ ? We will actually be interested solely in one case of this, although the general case is analogous; we ask, when can a Poisson fattening of type $(m, m)$ be extended to one of type $(m+1, m)$ ? This may be read off from the central extension

$$
\mathcal{O}\left(L_{\Sigma}^{-m}\right) \mapsto \mathscr{B}_{m+1, m} \rightarrow \mathscr{B}_{m, m} ;
$$

thus the obstruction to finding such an extension is an element of $H^{2}\left(\Sigma, \mathcal{O}\left(L_{\Sigma}^{-m}\right)\right)$. But this obstruction has the following property, which will be the key-stone of the proof of the main theorem.

Proposition 7.4. ("Localization Theorem"). Let $\Sigma \subset X$ be a Legendrian submanifold of a complex contact manifold, and let a Poisson thickening $X^{(m)}$ be a given Poisson 
thickening; let $\Sigma^{(m, m)}$ be the associated Poisson fattening of type $(m, m)$. Then the obstruction to extending $\Sigma^{(m, m)}$ to a Poisson fattening of type $(m+1, m)$ is obtained from the obstruction to extending $X^{(m)}$ to a Poisson thickening of order $m+1$ by applying the restriction map

$$
H^{2}\left(X, \mathcal{O}\left(L^{-m}\right)\right) \rightarrow H^{2}\left(\Sigma, \mathcal{O}\left(L_{\Sigma}^{-m}\right)\right)
$$

Proof. The obstruction to extending $X^{(m)}$ may be computed by arbitrarily extending the transition functions of $X^{(m)}$ to elements of $\mathscr{C}_{m+1}$ and then computing the incompatibility on triple overlaps. Similarly, the obstruction to extending a 1-cocycle for $\mathscr{B}_{m, m}$ to $\mathscr{B}_{m+1, m}$ and computing the incompatibility on triple overlaps. If, on a neighborhood of $\Sigma$, we take the transition functions for $X$ to be in $\mathscr{C}_{m} \Sigma$, extending them to $\mathscr{C}_{(m+1) \Sigma}$ induces an extension of their images in $\mathscr{B}_{m, m}$ to $\mathscr{B}_{m+1, m}$. The result follows. Q.E.D.

\section{Fattening the Sky}

Let $Q=\mathbb{P}_{1} \times \mathbb{P}_{1}$ be the 2-quadric, which will be our standard model of a sky $Q_{X} \subset \mathcal{N}$; we will study the behavior near $Q_{x}$ of an arbitrary ambitwistor space $\mathscr{N}$ by considering Legendrian fattenings of $Q$, and understand the obstructions to Poisson-thickening $\mathscr{N}$ by studying the obstructions to extending a Legendrian fattening to a Poisson fattening. Since the restriction of the contact line bundle to a sky is the $\mathcal{O}(1,1)$ line bundle (= the divisor of the diagonal $\Delta\left(\mathbb{P}_{1}\right) \subset \mathbb{P}_{1} \times \mathbb{P}_{1}$ ), we consider only Legendrian fattenings of $\Sigma=Q$ with fundamental line bundle $L_{\Sigma}=\mathcal{O}(1,1)$; and since the canonical first-order Poisson thickening is generated by $\log _{*}\left[L_{+}\right] \in H^{1}(\mathscr{N}, \mathcal{O} / \mathbb{C})$, we take the fundamental cohomology class $\alpha \in H^{1}(\Sigma, \mathcal{O} / \mathbb{C})$ to be the logarithm of the line bundle $\mathcal{O}(1,0)(=$ the divisor of the first factor $\mathbb{P}_{1} \times\{$ point $\left.\} \subset \mathbb{P}_{1} \times \mathbb{P}_{1}\right)$; i.e. $\alpha \in H^{1}(Q, \mathcal{O} / \mathbb{C})=H^{2}(Q, \mathbb{C})$ is the Poincare dual of the first factor. One may then check that

$$
\hat{N} \cong \mathcal{O}(1,0) \oplus \mathcal{O}(1,0) \oplus \mathcal{O}(0,1) \oplus \mathcal{O}(0,1),
$$

either by direct computation from the definition of Sect. 7 or by using Corollary 4.2. Indeed, we have natural identification

$$
\begin{aligned}
\hat{N} & =\left[\mathbf{S}_{+} \otimes \mathcal{O}(0,1)\right] \oplus\left[\mathbf{S}_{-} \otimes \mathcal{O}(1,0)\right] \\
& =\mathcal{O}^{A}(0,1) \oplus \mathcal{O}^{A^{\prime}}(1,0),
\end{aligned}
$$

where $Q=\mathbb{P}\left(\mathbf{S}_{+}^{*}\right) \times \mathbb{P}\left(\mathbf{S}_{-}^{*}\right)$ and where the abstract indices $A$ and $A^{\prime}$ correspond to the two 2-dimensional vector spaces $\mathbf{S}_{+}$and $\mathbf{S}_{-}$, respectively. $N$ is related to $\hat{N}$ by an exact sequence

$$
0 \rightarrow N \rightarrow \hat{N} \underset{w}{\rightarrow} \mathcal{O}(1,1) \rightarrow 0
$$

where

$$
\varpi\left(f^{A}, g^{A^{\prime}}\right)_{\mid\left(\mu_{A}, \pi_{A^{\prime}}\right)}=f^{A} \mu_{A}+g^{A^{\prime}} \pi_{A^{\prime}} .
$$

$N$ can also be described by the exact sequence

$$
0 \rightarrow \mathcal{O}(-1,-1)[-2] \rightarrow \mathcal{O}^{A A^{\prime}} \rightarrow N \rightarrow 0
$$


arising from the fact that it is generated by its global sections, or from the exact sequence

$$
0 \rightarrow \mathcal{O}(1,-1)[1] \oplus \mathcal{O}(-1,1)[1] \rightarrow N \rightarrow \mathcal{O}(1,1) \rightarrow 0
$$

corresponding to the fact that $N=J^{1} \mathcal{O}(1,1)$. (Numbers in square brackets are conformal weights.)

Let us now consider Legendrian fattenings of $Q$. The exact sequence

$$
0 \rightarrow N^{*} \rightarrow \mathcal{O}_{a} \rightarrow \mathcal{O}(1,1)[2] \rightarrow 0,
$$

where $a=A A^{\prime}$, gives rise to exact sequences

and hence

$$
0 \rightarrow \odot^{\ell} N^{*} \rightarrow \underbrace{\mathcal{O}_{(a \ldots b c)}}_{\ell} \rightarrow \underbrace{\mathcal{O}_{(a \ldots b)}}_{\ell-1}(1,1)[2] \rightarrow 0
$$

It follows that

$$
0 \rightarrow \mathcal{O}(1,1) \otimes \odot^{\ell+2} N^{*} \rightarrow \underbrace{\mathcal{O}_{(a \ldots b c)}}_{\ell+2}(1,1) \rightarrow \underbrace{\mathcal{O}_{(a \ldots b)}}_{\ell+1}(2,2)[2] \rightarrow 0 .
$$

$$
H^{2}\left(Q, \mathcal{O}(1,1) \otimes \bigodot^{\ell+2} N^{*}\right)=0
$$

for all $\ell$, and that

$$
\begin{array}{r}
H^{1}\left(Q, \mathcal{O}(1,1) \otimes \odot^{\ell+2} N^{*}\right)=\text { coker sym: } T \otimes \odot^{\ell+2} T^{*} \rightarrow\left(\odot^{\ell+1} T^{*}\right) \otimes\left(\odot_{0}^{2} T^{*}\right)[2] \\
\varphi_{(a \ldots b c)}{ }^{d} \mapsto \varphi_{a \ldots b(c d)},
\end{array}
$$

where $T=H^{1}(Q, \mathcal{O}(N))$ is the abstract tangent space of our space-time. Thus, there is no obstruction to extending a Legendrian fattening from order to order, while the freedom in so doing corresponds formally to choosing the partial derivatives of a conformal metric modulo the action of vector fields fixing our ideal point. Let us check that the above interpretation of the freedom is literally correct.

This is best seen by considering the following fact of independent interest: the natural Penrose-transform identification of $\Gamma\left(\mathscr{M}, \mathcal{O}\left(\odot_{0}^{2} T^{*} \mathscr{M}\right)[2]\right) / \Gamma(\mathscr{M}, \mathcal{O}(T M))$ and $H^{1}\left(\mathscr{N}_{1} \mathcal{O}(2)\right)$ agrees with the Kodaira-Spencer map from

$\Gamma\left(\mathscr{M}, \mathcal{O}\left(\odot_{0}^{2} T^{*} \mathscr{M}\right)[2]\right) / \Gamma(\mathscr{M}, \mathcal{O}(T \mathscr{M}))($ considered as infinitesimal deformations of the conformal metric, modulo infinitesimal biholomorphisms) to $H^{1}(\mathcal{N}, \mathcal{O}(L))$ (considered as the infinitesimal contact deformations) up to a constant factor. The claim will then follow by restriction of the action to the Legendrian fattenings of sky and induction on $\ell$, since the freedom of extending a Legendrian fattening to higher order only involves linear actions by derivations.

For this purpose, the most convenient picture of small deformations of conformal structure is the following: let $(\mathscr{M},[\mathrm{g}])$ be a complex conformal space-time, and, for each automorphism $\varphi \in \Gamma\left(\mathscr{M}, \mathcal{O}\left(T^{*} \mathscr{M} \otimes T \mathscr{M}\right)\right)$ of tangent bundle of $\mathscr{M}$, consider the new conformal structure $\left(\varphi^{-1}\right)^{*}[\mathrm{~g}]$. We in fact obtain all conformal structures near [g] by taking $\varphi=1+t A$, where $A$ is symmetric and trace-free: $A \in \Gamma\left(\mathscr{M}, \mathcal{O}\left(\odot^{2} T^{*} \mathscr{M}\right)[2]\right)$. (Indeed, the transformation $1+t A$ gives rise to the conformal structure $\left[\mathfrak{g}-2 t A+o\left(t^{2}\right)\right]$.) The advantage of this point of view is that the total space of the null-quadric bundle $\mathscr{Q} \rightarrow \mathscr{M}$ remains the same, but the degenerate contact structure induced on it by $\mathbb{P}\left(T^{*} \mathscr{M}\right)$ varies, as does, consequently, the induced foliation of $\mathscr{Q}$ by the directions of degeneracy of this structure, thus deforming the quotient contact manifold $\mathscr{N}$. To be precise, the 
contact form

$$
\Theta=p_{a} d x^{a}
$$

of $\mathbb{P}\left(T^{*} \mathscr{M}\right)$ is deformed to

$$
\Theta_{t}=\ominus+t A_{a}^{b} p_{b} d x^{a}
$$

by the action of $\varphi=1+t A$. Therefore, local isomorphisms between the degenerate contact manifolds $\left(\mathscr{Q}, \Theta_{t}\right)$ and $(\mathscr{Q}, \Theta)$ are generated, to first order in $t$, by local vector fields $v_{\alpha}$ on the (non-projective) null cone $\widehat{\mathscr{Q}} \subset T^{*} \mathscr{M}$ satisfying

$$
\mathscr{L}_{v_{\alpha}} \omega=d \Psi(A)
$$

where $\omega=d p_{a} \wedge d x^{a}$ and $\Psi(A)=A_{a}{ }^{b} p_{b} d x^{a}$. Thus $\left.d \Psi=d\left(v_{\alpha}\right\lrcorner \omega\right)$, and $\left.\Psi=v_{\alpha}\right\lrcorner \omega+d h_{\alpha}$ for some local system of functions $h_{\alpha}$. These functions may be explicitly found by choosing a system of local system of transversals to the fibers of the MarsdenWeinstein symplectic quotient map $\hat{q}: \hat{\mathscr{Q}} \rightarrow \hat{\mathscr{N}}$, where $\hat{\mathscr{N}}$ is the total space of the inverse contact line-bundle $L^{*} \rightarrow \mathscr{N}$. Indeed, letting $S_{\alpha}: U_{\alpha} \rightarrow \widehat{\mathscr{Q}}$ be a system of local sections of $\hat{q}$ for some covering $U_{\alpha}$ of $\hat{\mathcal{N}}$, we may set

But

$$
h_{\alpha}(p)=\int_{S_{\alpha}(q(p))}^{p}\left[\left.\Psi(A)\right|_{q^{-1}(q(p))}\right]
$$

$$
\left.\left(v_{\alpha}-v_{\beta}\right)\right\lrcorner \omega=d\left(h_{\beta}-h_{\alpha}\right)
$$

and so

$$
\begin{aligned}
\Theta\left(v_{\alpha}-v_{\beta}\right) & \left.=\omega\left(\xi, v_{\alpha}-v_{\beta}\right)=\xi\right\lrcorner d\left(h_{\alpha}-h_{\beta}\right)=\xi\left(h_{\alpha}-h_{\beta}\right) \\
& =\xi \int_{S_{\alpha}}^{S_{\beta}}\left[\left.\Psi(A)\right|_{\text {fibers of } q}\right],
\end{aligned}
$$
where $\xi$ is the Euler vector field $p_{a} \frac{\partial}{\partial p_{a}}$. Taking our sections $S_{\alpha}$ to be $(\mathbb{C}-\{0\})$ -
invariant, this simplifies to become

$$
\Theta\left(v_{\alpha}-v_{\beta}\right)=\int_{S_{\alpha}}^{s_{\beta}}\left[\left.\Psi(A)\right|_{\text {fibers of } \hat{q}}\right]
$$

because $\Psi(A)$ has homogeneity 1 . Now $\Theta\left(v_{\alpha}-v_{\beta}\right)$ is exactly the section of $L \rightarrow \mathcal{N}$ which generates the contact vector field $q_{*}\left(v_{\alpha}-v_{\beta}\right)$, so that $\Theta\left(v_{\alpha}-v_{\beta}\right)$ is a cocycle representing the image of $A$ under the Kodaira-Spencer map. Since $s_{\beta}$

$\int_{S_{\alpha}}^{S_{\beta}}\left[\left.\Psi(A)\right|_{\text {fibers of } \hat{q}}\right]$ depends linearly on $A$, this shows that the Kodaira-Spencer map from the linearized deformations of the conformal metric to $H^{1}(\mathcal{N}, \mathcal{O}(L))$ is linear. Indeed, we also see quite directly that it is an isomorphism, since $H^{1}(\mathcal{N}, \mathcal{O}(L))=$ $H^{1}\left(\mathscr{Q}, q^{-1} \mathcal{O}(L)\right)$, and we have the exact sequence

$$
0 \rightarrow q^{-1} \mathcal{O}(L) \rightarrow \mathcal{O}(1,1) \underset{d_{q}}{\longrightarrow} \Omega_{q}^{1}(1,1) \rightarrow 0 ;
$$

our Kodaira-Spencer map from $\Gamma\left(\mathscr{M}, \mathcal{O}\left(\odot_{0}^{2} T^{*} \mathscr{M}\right)[2]\right)=H^{0}\left(\mathscr{Q}, \Omega_{q}^{1}(1,1)\right)$ to 
$H^{1}(\mathcal{N}, \mathcal{O}(L))=H^{1}\left(\mathscr{Q}, q^{-1} \mathcal{O}(L)\right)$, given by

$$
A_{a}{ }^{b} \mapsto\left[\int_{S_{\alpha}}^{S_{\beta}}\left(\left.A_{a}^{b} p_{b} d x^{a}\right|_{\text {fibers }}\right)\right],
$$

is nothing but the Bockstein operator

$$
\cdots \rightarrow H^{0}\left(\mathscr{Q}, \Omega_{q}^{1}(1,1)\right) \rightarrow H^{1}\left(\mathscr{Q}, q^{-1} \mathcal{O}(L)\right) \rightarrow 0
$$

obtained on Čech cohomology by introducing local splittings for the map $d_{q}: \mathcal{O}(1,1) \rightarrow \Omega_{q}^{1}(1,1)$ which differentiates up the fibers of $q$. Using the same formula for the Kodaira-Spencer map, we also see that changing the $(\ell+1)$-st derivatives of the metric at some $x \in \mathscr{M}$ results in a linearly related change in the corresponding $(\ell+1)$-st order Legendrian fattening, as parametrized by $H^{1}\left(Q, \mathcal{O}(1,1) \otimes \odot^{\ell+2} N^{*}\right)$. Indeed, this Kodaira-Spencer map for Legendrian fattenings must, by the above argument, be induced by the Penrose transform, and is therefore exactly our original Bockstein operator

$$
H^{0}\left(Q, \mathcal{O}_{(a \ldots b)}(2,2)[2]\right) \rightarrow H^{1}\left(Q, \mathcal{O}(1,1) \otimes \bigodot^{\ell+2} N^{*}\right),
$$

this being, after all, just the $(\ell+1)$-st term in the Taylor expansion of the Bockstein operator

$$
H^{0}\left(\mathscr{Q}, \Omega_{q}^{1}(1,1)\right) \rightarrow H^{1}\left(\mathscr{Q}, q^{-1} \mathcal{O}(L)\right)
$$

around a fiber of $\mathscr{Q} \rightarrow \mathscr{M}$. We have therefore proved

Proposition 8.1. The set of Legendrian fattenings of order $\ell$ of $Q$ with $L_{\Sigma}=\mathcal{O}(1,1)$ corresponds exactly to the set of $\ell$-jets of conformal metrics at a point modulo biholomorphisms. Moreover, this identification is compatible with the action of $\left(\left(\odot^{\ell} T^{*}\right) \otimes \odot_{0}^{2} T^{*}[2]\right) /\left(\odot^{\ell-1} T^{*}\right) \otimes T$ on these two sets.

Let us now make some general observations on the structure of such Kodaira-Spencer maps. Suppose for a moment that $Z$ is the leaf space of a holomorphic foliation of $Y$ by simply connected curves; let $q: Y \rightarrow Z$ denote the canonical projection. If we deform the foliation, we induce a deformation of $Z$. Such a deformation of the foliation amounts to a deformation of the corresponding 1-dimensional sub-bundle of the tangent bundle, and at the infinitesimal level is specified by a section $\chi$ of $\left(q^{*} T Z\right) \otimes \Omega_{q}^{1}$. Cover $Z$ by open sets $\left\{U_{\alpha}\right\}$ on which we may find sections $s_{\alpha}: U_{\alpha} \rightarrow Y$ of $q$. When we deform our foliation, a deformation of $Z$ is induced by identifying $x_{\alpha} \in U_{\alpha}$ with $x_{\beta} \in U_{\beta}$ if $s_{\alpha}\left(x_{\alpha}\right)$ and $s_{\beta}\left(x_{\beta}\right)$ are on the same leaf of the new foliation. On the infinitesimal level, this means that the transition functions of $Z$ are to be altered by flowing along the vector field

$$
V_{\alpha \beta}=\left.\int_{S_{\alpha}}^{S_{\beta}} \chi\right|_{\text {fibers of } q} .
$$

This shows that the Kodaira-Spencer map from infinitesimal changes in the foliation to $H^{1}(Z, \mathcal{O}(T Z))$ is given by the Bockstein operator $H^{1}(Z, O(T Z)$ )

$$
\cdots \rightarrow H^{0}\left(Y, \Omega_{q}^{1}\left(q^{*} T Z\right)\right) \rightarrow H^{1}\left(Y, q^{-1} \mathcal{O}(T Z)\right) \rightarrow \cdots
$$


of the induced by the short exact sequence

$$
0 \rightarrow q^{-1} \mathcal{O}(T Z) \rightarrow \mathcal{O}\left(q^{*} T Z\right) \stackrel{d_{q}}{\rightarrow} \Omega_{q}^{1}\left(q^{*} T Z\right) \rightarrow 0 .
$$

The same procedure also works in the case of leaves of higher dimension. Due to the Frobenius integrability condition, however, $\Omega_{q}^{1}\left(q^{*} T Z\right)$ is then replaced by

$$
K=\operatorname{ker} d_{q}: \Omega^{1}\left(q^{*} T Z\right) \rightarrow \Omega^{2}\left(q^{*} T Z\right),
$$

so that the short exact sequence becomes

$$
0 \rightarrow q^{-1} \mathcal{O}(T Z) \rightarrow \mathcal{O}\left(q^{*} T Z\right) \stackrel{d_{q}}{\rightarrow} K \rightarrow 0
$$

and our Bockstein operator is

$$
\delta: H^{0}(Y, K) \rightarrow H^{1}\left(Y, q^{-1} \mathcal{O}(T Z)\right) .
$$

A particular case of the above occurs when $Y$ has a $(\mathbb{C}-\{0\})$-action commuting with $q$, so that we have a diagram

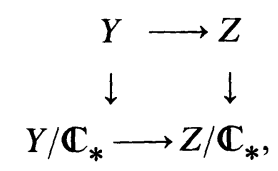

where we will assume that $Z \rightarrow Z / \mathbb{C}^{*}$ is a principal $(\mathbb{C}-\{0\})$-bundle. If we deform the foliation of our principal line bundle $Y \rightarrow Y / \mathbb{C}_{*}$ through $\mathbb{C}_{*}$-invariant foliations, we induce a deformation of the line bundle $Z \rightarrow Z / \mathbb{C}_{*}$ through line-bundles over variable base spaces. If $\hat{\Theta}$ is the sheaf on $Z / \mathbb{C}_{*}$ of $\mathbb{C}_{*}$-invariant vector fields on $Z$, the corresponding Kodaira-Spencer map is just the Bockstein operator

$$
H^{0}\left(Y / \mathbb{C}_{*}, \Omega_{q}^{1} \otimes q^{*} \hat{\theta}\right) \rightarrow H^{1}\left(Y / \mathbb{C}_{*}, q^{-1} \hat{\theta}\right)
$$

of the short exact sequence

$$
0 \rightarrow q^{-1} \hat{\theta} \rightarrow \mathcal{O}\left(q^{*} T Z\right)^{d_{q}} \rightarrow \Omega_{q}^{1} \otimes q^{*} \hat{\theta} \rightarrow 0 .
$$

Let us now consider what happens to the "spinor" line bundle $L_{+} \rightarrow \mathscr{N}$ as we deform a conformal metric; we begin by considering the Kodaira-Spencer map

$$
\Gamma\left(\mathscr{M}, \mathcal{O}\left(\odot_{0}^{2} T^{*} \mathscr{M}\right)[2]\right) \rightarrow H^{1}(\mathscr{N}, \hat{\Theta}),
$$

where

$$
\hat{\Theta}=\left(L_{+} \otimes J^{1} L_{+}^{*}\right)^{*}=\left(L_{+}^{*} \otimes J^{1} L_{+}\right)^{*}
$$

is the sheaf on $\mathscr{N}$ of $\mathbb{C}_{*}$-invariant vector fields on $L_{+}-0_{L_{+}}=L_{+}^{*}-0_{L_{+}^{*}}$. As always, our analysis proceeds via the double fibration

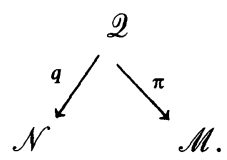

We will identify $\mathscr{Q}$ with $\mathbb{P}\left(\boldsymbol{S}_{+}\right) \oplus \mathbb{P}\left(\boldsymbol{S}_{-}\right) \rightarrow \mathscr{M}$, and the projection $q^{*}\left(L_{+}^{*}-0_{L_{+}^{*}}\right) \rightarrow \mathscr{Q}$ then becomes

$$
\left(\mathbf{S}_{+}-0_{\mathbf{S}_{+}}\right) \oplus \mathbb{P}\left(\mathbf{S}_{-}\right) \rightarrow \mathbb{P}\left(\mathbf{S}_{+}\right) \oplus \mathbb{P}\left(\mathbf{S}_{-}\right)
$$


The foliation of $\left(\mathbf{S}_{+}-0_{\mathbf{S}_{+}}\right) \oplus \mathbb{P}\left(\mathbf{S}_{-}\right)$which gives rise to $L_{+}^{*}-0_{L_{+}^{*}}$ is then the image of the foliation of $\left(\mathbf{S}_{+}-0_{\mathbf{S}_{+}}\right) \oplus\left(\mathbf{S}_{-}-0_{\mathbf{S}_{-}}\right)$tangent to the vector field $\eta$ whose value at $\left(\lambda^{A}, \pi^{A^{\prime}}\right)$ is the horizontal lift of $\lambda^{A} \pi^{A^{\prime}} \in T \mathscr{M}$ via the metric connection of any metric $g$ in the fixed conformal class. Now when we fix our conformal structure, we have a fixed isomorphism

$$
\sigma: \mathbf{S}_{+} \otimes \mathbf{S}_{-} \rightarrow T \mathscr{M}
$$

if we vary this isomorphism, the effect is precisely to vary the conformal structure. Using our previous formalism, the endomorphism $1+t A$ of $T \mathscr{M}$ changes $\sigma$ to $\sigma_{t}=\sigma+t A \circ \sigma$ and changes the metric to

$$
\mathfrak{g}_{t}=\mathfrak{g}-2 t A+O\left(t^{2}\right) .
$$

The metric connection consequently varies as

$$
\nabla_{t}=\nabla+t B+O\left(t^{2}\right)
$$

where

$$
B_{K \ell}^{j}=-2 \nabla_{(K} A_{\ell)}^{j}+\nabla^{j} A_{K \ell},
$$

but we must pull this back via the "gauge transformation" $1+t A$ in order to see how this acts on $\boldsymbol{S}_{+} \oplus \boldsymbol{S}_{-}$, considered as a fixed bundle. The corresponding gauge-transformed connections are then given by

where

$$
\hat{\nabla}_{t}=\nabla+t C+O\left(t^{2}\right)
$$

$$
C_{k \ell}^{j}=\nabla^{j} A_{k \ell}-\nabla_{\ell} A_{k}^{j} .
$$

This gives as a family of $S O(4, \mathbb{C})$ connections on $T \mathscr{M}$, and the induced family of connections on $\boldsymbol{S}_{+}$are given by

$$
\hat{\nabla}_{t}=\nabla+t \hat{C}+O\left(t^{2}\right),
$$

where $\hat{C}_{k L}^{j}=\frac{1}{2} C_{k L J^{\prime}}^{J J^{\prime}}$, while for $\boldsymbol{S}_{\text {- we have }}$

$$
\nabla_{t}=\nabla+t \hat{C}^{\prime}+O\left(t^{2}\right)
$$

where $\left(\hat{C}^{\prime}\right)_{k}{ }^{J^{\prime}}{ }_{L^{\prime}}=\frac{1}{2} C_{k}^{J J^{\prime}}{ }{ }_{L^{\prime}}$. The corresponding foliation of $\left(\mathbf{S}_{+}-0_{\mathbf{S}^{+}}\right) \oplus\left(\mathbf{S}_{-}-0_{\mathbf{S}_{-}}\right)$ are therefore tangent to

$$
\begin{aligned}
\eta_{t}= & \eta+t A_{J J^{\prime}}{ }^{K K^{\prime}} \lambda^{J} \pi^{J^{\prime}} \frac{\hat{\partial}}{\partial x^{K K^{\prime}}}+\frac{1}{2} t \lambda^{J} \lambda^{K} \pi^{J^{\prime}}\left(\nabla_{K K^{\prime}} A_{J J^{\prime}}{ }^{L K^{\prime}}-\nabla^{L K^{\prime}} A_{J J^{\prime} K K^{\prime}}\right) \frac{\partial}{\partial \lambda^{L}} \\
& +\frac{1}{2} t \lambda^{J} \pi^{J^{\prime}} \pi^{K^{\prime}}\left(\nabla_{K K^{\prime}} A_{J J^{\prime}}{ }^{L K^{\prime}}-\nabla^{K L^{\prime}} A_{J J^{\prime} K K^{\prime}}\right) \frac{\partial}{\partial \pi^{L^{\prime}}}
\end{aligned}
$$

where $\frac{\hat{\partial}}{\partial x^{K K^{\prime}}}$ is used to denote the isomorphism $\sigma: \mathbf{S}_{+} \otimes \mathbf{S}_{-} \rightarrow T \mathscr{M}$ lifted to the horizontal space $H \cong T \mathscr{M}$ of $\nabla$, and where $\frac{\partial}{\partial \pi}$ and $\frac{\partial}{\partial \lambda}$ are used to indicate vertical vector fields on $\mathbf{S}_{+} \oplus \mathbf{S}_{-}$. This shows that the foliation-variation 1-form

$$
\chi(A) \in \Gamma\left(Q, \Omega_{q}^{1}\left(q^{*} \hat{\theta}\right)\right)
$$

is given in terms of A by an invariant first-order linear differential operator. In 
particular, the infinitesimal version of our procedure of assigning a line-bundle $L_{+} \rightarrow \mathscr{N}$ to every ambi-twistor space gives rise to a linear map

$$
H^{1}(\mathcal{N}, \mathcal{O}(L)) \rightarrow H^{1}(\mathcal{N}, \hat{\theta})
$$

making the diagram

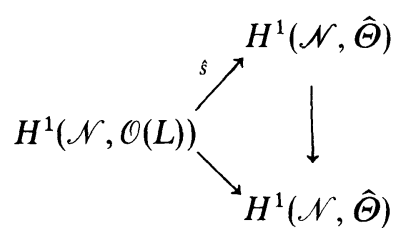

commute.

Now the contact structure gives a splitting of the exact sequence

$$
0 \rightarrow \mathcal{O}(D) \rightarrow \Theta \stackrel{\ominus}{\rightarrow} \mathcal{O}(L) \rightarrow 0,
$$

so that we have an exact sequence

$$
0 \rightarrow \hat{\mathscr{C}} \rightarrow \hat{\Theta} \rightarrow \mathcal{O}(D) \rightarrow 0
$$

where, as in Sect. $4, \hat{\mathscr{C}}$ is the sheaf of $(\mathbb{C}-\{0\})$-invariant vector fields on $L_{+} \rightarrow \mathscr{N}$ which cover infinitesimal contact transformations. But the contact structure on $\mathscr{N}$ is unique, so $H^{0}(\mathcal{N}, \mathcal{O}(D))=0$; this is because $H^{0}(\mathscr{N}, \mathcal{O}(D))$ is the kernel of $H^{0}(\mathscr{N}, \mathcal{O}(T \mathscr{N})) \rightarrow H^{0}(\mathscr{N}, \mathcal{O}(L))$, while this map must be an isomorphism because every automorphism of $\mathscr{N}$ must be a contact transformation. Hence we have an exact sequence

$$
0 \rightarrow H^{1}(\mathscr{N}, \widehat{\mathscr{C}}) \rightarrow H^{1}(\mathscr{N}, \hat{\Theta}) \rightarrow \cdots
$$

so that we may deduce that the natural map $H^{1}(\mathcal{N}, \mathcal{O}(L)) \rightarrow H^{1}(\mathscr{N}, \hat{\mathscr{C}})$ is actually linear, since we have the commutative diagram

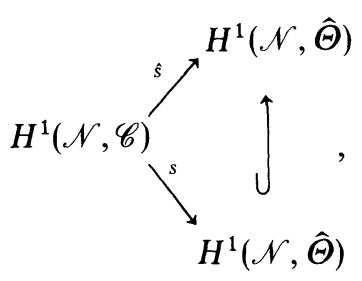

where $\hat{s}$ is now known to be linear. Thus the procedure of assigning a "spinor" line-bundle $L_{+}$to every ambitwistor space corresponds on the linearized level to a linear splitting $s$ of the natural projection $H^{1}(\mathscr{N}, \widehat{\mathscr{C}}) \rightarrow H^{1}(\mathcal{N}, \mathscr{C})$. But this is then related to the Kodaira-Spencer map for first-order Poisson thickenings by Proposition 4.2, from which we immediately deduce that our assignment of a canonical manner in which the $\ell$-th fattening of a sky varies as we vary the conformal metric through metrics which agree to order $\ell-1$ at our base point. Since the splitting $s$ corresponds via the Penrose transform to an invariant linear first-order differential operator, it follows that we have a corresponding linear splitting of the restriction map on fattening freedoms: 
Proposition 8.2. The canonical thickening procedure corresponds to an SO(4)equivariant linear splitting of the restriction map

$$
H^{1}\left(Q, L_{\Sigma} \otimes\left(\bigodot^{\ell+1} \hat{N}^{*} / \bigodot^{\ell-1} \hat{N}^{*}\right)\right) \rightarrow H^{1}\left(Q, L_{\Sigma} \otimes \bigodot^{\ell+1} N^{*}\right)
$$

for freedom in Poisson fattening.

\section{Calculating the Obstructions}

We begin by showing that there is no obstruction to extending the canonical first-order Poisson thickening of any ambi-twistor space $\mathscr{N}$ to fourth order. By Proposition 7.4 this amounts to a problem of extending the type $(3,3)$ Poisson fattening of every sky (induced by $\mathcal{N}^{(3)}$ ) to a fattening of type $(4,3)$.

Let a type $(3,2)$ Poisson fattening of $Q=Q_{x}$ be given. Associated with this fattening is a second order Legendrian fattening of $Q$, and we know by the calculations of the previous section that this Legendrian fattening may be extended to third order. The freedom in so doing is given by $H^{1}\left(Q, \mathcal{O}(1,1) \otimes \odot^{4} N^{*}\right)$. On the other hand, we might simply attempt to extend our fattening of type $(3,2)$ (=type $(4,2))$ to a fattening of type $(4,3)$. There is no obstruction to doing so, because

$$
\begin{aligned}
H^{2}\left(Q, \mathcal{O}(1,1) \otimes \odot^{4} \hat{N}^{*}\right) & =H^{1}\left(\mathbb{P}_{1} \times \mathbb{P}_{1}, \mathcal{O}(1,1) \otimes \odot^{4}\left[\mathcal{O}_{A}(-1,0) \otimes \mathcal{O}_{A^{\prime}}(-1,0)\right]\right) \\
& =\bigoplus_{p+q=4} H^{1}(\mathbb{P}_{1} \times \mathbb{P}_{1}, \underbrace{(\mathcal{O}_{(A \cdots B)(} \underbrace{\left.A^{\prime} \cdots B^{\prime}\right)}_{q}}_{p}(1-q, 1-p)) \\
& =0 ;
\end{aligned}
$$

the freedom in so doing is given by $H^{1}\left(Q, \mathcal{O}(1,1) \otimes \odot^{4} \hat{N}^{*}\right)$, and is related to the previously mentioned Legendrian freedom by the long exact sequence

$\cdots \rightarrow H^{1}\left(\odot^{3} \hat{N}^{*}\right) \rightarrow H^{1}\left(\mathcal{O}(1,1) \otimes \odot{ }^{4} \hat{N}^{*}\right) \rightarrow H^{1}\left(\mathcal{O}(1,1) \otimes \odot^{4} N^{*}\right) \rightarrow H^{2}\left(\odot^{3} \hat{N}^{+}\right) \rightarrow \cdots$. Since

$$
\begin{aligned}
H^{2}\left(Q, \odot^{3} \hat{N}^{*}\right) & =\bigoplus_{p+q=3} H^{2}(\mathbb{P}_{1} \times \mathbb{P}_{1}, \underbrace{\mathcal{O}_{(A \cdots B)}}_{p} \underbrace{\left(A^{\prime} \cdots B^{\prime}\right)}_{q}(-q,-p)) \\
& =0,
\end{aligned}
$$

it follows that every third order Legendrian fattening of $Q$ extends as a Poisson fattening of type $(4,3)$ agreeing with the given fattening of type $(3,2)$.

Assuming that our Poisson fattening of type $(3,2)$ was induced by the canonical thickening $\mathscr{N}^{(3)}$ by taking $Q$ to be identified with a sky $Q_{x}$, we must now show that among the various Poisson fattening of type $(4,3)$ that extend the given one there is one that not only extends the third order Legendrian fattening of $Q_{x} \subset \mathcal{N}$, but also extends the Poisson fattening of type $(1,3)$ induced by the canonical Poisson thickening $\mathscr{N}^{(1)}$. The freedom in extending the induced fattening of type $(1,2)$ to type $(1,3)$ is given by

$$
H^{1}\left(Q,\left[\mathcal{O}(1,1) \otimes \odot^{4} \hat{N}^{*}\right] /\left[\mathcal{O}(-1,-1) \otimes \odot^{2} \hat{N}^{*}\right]\right),
$$

and is related to the Legendrian freedom by the exact sequence

$$
H^{1}\left(\odot^{3} N^{*}\right) \rightarrow H^{1}\left(\mathcal{O}(1,1) \otimes \odot^{4} \hat{N}^{*} / \mathcal{O}(-1,1) \otimes \bigodot^{2} \hat{N}^{*}\right) \rightarrow H^{1}\left(\mathcal{O}(1,1) \otimes \odot^{4} N^{*}\right) .
$$

Our assignment of a first-order thickening to every ambitwistor space gives an 
$S O(4)$-equivariant splitting of the above sequence. Now consider the exact sequence

$$
\begin{aligned}
\cdots \rightarrow H^{1}\left(\mathcal{O}(1,1) \otimes \odot^{4} \hat{N}^{*}\right) & \rightarrow H^{1}(\mathcal{O}(1,1) \otimes \\
& \left.\odot^{4} N^{*} / \mathcal{O}(-1,-1) \otimes \odot^{2} \hat{N}^{*}\right) \\
& \rightarrow H^{2}\left(\mathcal{O}(-1,-1) \otimes \odot^{2} \hat{N}^{*}\right) \rightarrow \cdots,
\end{aligned}
$$

which relates the freedoms for fattenings of type $(4,3)$ and $(1,3)$. Composing the Bockstein operator of the latter sequence with the splitting of the former sequence, we obtain an $S O(4)$-equivariant linear map

$$
H^{1}\left(Q, \mathcal{O}(1,1) \otimes \odot^{4} N^{*}\right) \rightarrow H^{2}\left(Q, \mathcal{O}(-1,-1) \otimes \bigodot^{2} \hat{N}^{*}\right),
$$

corresponding to an affine map from the space of 3-jets of conformal metrics extending a given 2-jet to the space of 3-forms; but since

$$
H^{1}\left(Q, \mathcal{O}(1,1) \otimes \odot{ }^{4} N^{*}\right)=\mathbb{C}_{(A B C D E) E^{\prime}} \oplus \mathbb{C}_{(A B C) E^{\prime}}[-1] \oplus \mathbb{C}_{\left(A^{\prime} B^{\prime} C^{\prime} D^{\prime} E^{\prime}\right) E} \oplus \mathbb{C}_{\left(A^{\prime} B^{\prime} C^{\prime}\right) E}[-1]
$$

and

$$
H^{2}\left(Q, \mathcal{O}(-1,-1) \otimes \odot^{2} \hat{N}^{*}\right)=\mathbb{C}_{A A^{\prime}}[-2]
$$

have no irreducible components in common, such a map must vanish identically. Thus there is a type $(4,3)$ Poisson thickening which agrees with the canonical choices of type $(3,2)$ and type $(2,3)$.

Now the canonically induced Poisson thickening of type $(3,3)$ is the only one of this type that extends the induced fattenings of type $(3,2)$ and $(2,3)$, since we have the exact sequence

$$
\begin{aligned}
\cdots & \rightarrow H^{1}\left(\mathcal{O}(-1,-1) \otimes \odot^{2} \hat{N}^{*} / \mathcal{O}(-3,-3)\right) \rightarrow H^{1}\left(\mathcal{O}(1,1) \otimes \odot^{4} \hat{N}^{*} / \mathcal{O}(-3,-3)\right) \\
& \rightarrow H^{1}\left(\mathcal{O}(1,1) \otimes \odot^{4} \hat{N}^{*} / \mathcal{O}(-1,-1) \otimes \odot^{2} \hat{N}^{*}\right),
\end{aligned}
$$

and

$$
H^{1}\left(Q, \mathcal{O}(-1,-1) \otimes \odot^{2} \hat{N}^{*} / \mathcal{O}(-3,-3)\right)=0 .
$$

Thus the canonically induced Poisson fattening of type $(3,3)$ extends to type $(4,3)$. Invoking Proposition 7.4 and the fact that elements of $H^{2}\left(\mathcal{N}, \mathcal{O}\left(L^{-3}\right)\right)$ are characterized by integration over skies, we therefore have

Theorem 9.1. The canonical first order Poisson thickening of any ambitwistor space extends uniquely to fourth order.

Remark. The same machinery may also be used to verify that the obstruction to extending a first order Poisson thickening derived from $L_{+} \otimes E, c_{1}(E)=0$, is precisely the electromagnetic current $\nabla^{a} F_{a b}$ of the connection associated to $E$ by the Ward correspondence. However, we will not need this fact, and so leave it as an exercise for the interested reader.

Let us turn now to the obstruction to existence of $\mathscr{N}^{(5)}$. We proceed by supposing we are given the type $(4,3)$ Poisson fatening of a sky $Q_{x}$ induced by the previously constructed canonical Poisson thickening $\mathscr{N}^{(4)}$ of our ambitwistor space. Again, there is no obstruction to extending it as a Poisson fattening of type $(5,4)$, since

$$
\begin{aligned}
H^{2}\left(Q, \mathcal{O}(1,1) \otimes \odot{ }^{5} \hat{N}^{*}\right) & =\bigoplus_{p+q=5} H^{2}(\mathbb{P}_{1} \times \mathbb{P}_{1}, \underbrace{\mathcal{O}_{(A \cdots B)}}_{p} \underbrace{\left.A^{\prime} \cdots B^{\prime}\right)}_{q}(1-q, 1-p)) \\
& =0 ;
\end{aligned}
$$


the freedom in so doing is given by $H^{1}\left(Q, \mathcal{O}(1,1) \otimes \odot^{5} \hat{N}^{*}\right)$. On the other hand, there is, as always, no obstruction to extending the associated Legendrian fattening to order 4, and the freedom of extension is given by $H^{1}\left(Q, \mathcal{O}(1,1) \otimes \odot^{5} N^{*}\right)$. The relation between these two kinds of freedom is read off from the exact sequence

$$
\cdots \rightarrow H^{1}\left(Q, \mathcal{O}(1,1) \otimes \odot^{5} \hat{N}^{*}\right) \rightarrow H^{1}\left(Q, \mathcal{O}(1,1) \otimes \bigodot^{5} N^{*}\right) \rightarrow H^{2}\left(Q, \odot^{4} \hat{N}^{*}\right) \rightarrow 0,
$$

where we have used the previously noted fact that $H^{2}\left(Q, \mathcal{O}(1,1) \otimes \odot^{5} \hat{N}^{*}\right)=0$. However,

$$
\begin{aligned}
H^{2}\left(Q, \bigodot^{4} \hat{N}^{*}\right) & =\bigoplus_{p+q=4} H^{2}\left(\mathbb{P}_{1} \times \mathbb{P}_{1}, \mathcal{O}_{(A \cdots B)\left(A^{\prime} \cdots B^{\prime}\right)}(-q,-p)\right) \\
& =\mathbb{C}_{(A B)\left(A^{\prime} B^{\prime}\right)}[2] \\
& =\left(\bigodot_{0}^{2} T^{*}\right)[-2],
\end{aligned}
$$

so we have a non-trivial obstruction of finding any Poisson fattening of type $(5,4)$ agreeing with our given Legendrian fattening of order 4 and Poisson fattening of type $(4,3)$; this obstruction is affine in fourth derivatives of the metric, and gives a surjection onto $\left(\odot_{0}^{2} T^{*}\right)[-2]$ which is $[(\mathbb{C}-\{0\}) \times S O(4)]$-equivariant.

Before we identify this obstruction in geometric terms, let us notice that it precisely gives the obstruction to extending the canonical Poisson fattening of type $(4,4)$ to type $(5,4)$. Indeed, our canonical choice of first-order thickenings corresponds to a linear splitting of

$$
H^{1}\left(Q, \mathcal{O}(1,1) \otimes \odot^{5} \hat{N}^{*} / \mathcal{O}(-1,-1) \otimes \odot^{3} \hat{N}^{*}\right) \rightarrow H^{1}\left(Q, \mathcal{O}(1,1) \otimes \odot{ }^{5} N^{*}\right),
$$

while the freedom in extending our type $(4,3)$ Poisson thickening to type $(4,4)$ in a manner consistent with our type $(2,4)$ Poisson thickening (specified by the splitting) is given by

$$
H^{1}\left(Q, \mathcal{O}(-1,-1) \otimes \odot^{3} \hat{N}^{*} / \mathcal{O}(-4,-4)\right)=0 .
$$

Thus the freedom in extending from $[(4,3),(0,4)]$ directly to $(5,4)$ is related to that of extending from $[(4,3),(4,4)]$ to $(5,4)$ via a commutative diagram

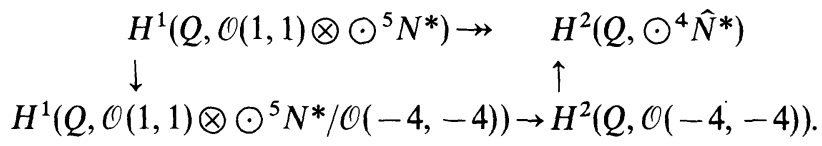

Therefore the relationship between these two kinds of freedom is given by an SO(4)-equivariant surjection

$$
H^{2}(\mathcal{O}(-4,-4)) \rightarrow H^{2}\left(\odot^{4} \hat{N}^{*}\right)
$$

between two irreducible $S O(4)$-module:

$$
H^{2}(Q, \mathcal{O}(-4,-4)) \cong H^{2}\left(Q, \odot{ }^{4} \hat{N}^{*}\right) \cong\left[\odot_{0}^{2} T^{*}\right][-2] .
$$

By Schur's lemma, this map is an isomorphism.

We now know that the obstruction depends upon four derivatives of the metric, and that its dependence on the fourth derivative is given by the Bockstein operator of the exact sequence 


$$
\cdots \rightarrow H^{1}\left(\odot^{4} \hat{N}^{*}\right) \rightarrow H^{1}\left(\odot^{4} \hat{N}^{*}(1,1)\right) \rightarrow H^{1}\left(\odot^{4} \hat{N}^{*}(1,1)\right) \rightarrow H^{2}\left(\odot^{4} \hat{N}^{*}\right) .
$$

The kernel of this map is therefore

$$
H^{1}\left(Q, \odot^{4} \hat{N}^{*}(1,1) / H^{1}\left(Q, \odot^{4} \hat{N}^{*}\right)=\mathbb{C}_{\left(A^{\prime} B^{\prime} C^{\prime} D^{\prime} E^{\prime}\right) F^{\prime}}^{(A)^{\prime}}[-2] \oplus \mathbb{C}_{(A B C D E) F}^{\left(A^{\prime} B^{\prime}\right)}[-2],\right.
$$

which has no irreducible component isomorphic to $\mathbb{C}_{(A B)\left(A^{\prime} B^{\prime}\right)}[-2]$. Consequently, the symbol of the obstruction is uniquely determined up to scale by the fact that it is $S O(4)$-equivariant. Since the dependence of the Bach tensor

$$
B_{a b}=\left(\nabla^{c} \nabla^{d}-\frac{1}{2} R^{c d}\right) C_{a c b d}
$$

upon the fourth derivative of $g_{a b}$ provides such a homomorphism, we conclude that the obstruction is

$$
B_{a b}+(\text { lower order terms) }
$$

times a non-zero universal constant determined by conventions for the Penrose transform.

These lower order terms, however, must vanish. Indeed, given a 3-jet of a metric, we can extend it arbitrarily to a 4-jet with vanishing Bach tensor, and then consider the obstruction element to which this gives rise; by the above argument, this is independent of choice, and so defines a map

$$
H^{1}\left(Q, \mathscr{S}_{1} / \mathscr{S}_{3}\right) \rightarrow H^{2}(Q, \mathcal{O}(-4,-4)) \text {. }
$$

Composing with the map from metrics to Legendrian fattenings, this gives us a holomorphic map $h$ from the set of 3-jets of metrics to $\bigodot_{0}^{2} T^{*}[-2]$. Thus $h$ assigns to every metric of the form

$$
g_{a b}=\delta_{a b}+r_{(a b)(c d)} x^{c} x^{d}+s_{(a b)(c d e)} x^{c} x^{d} x^{e}
$$

a tensor trace-free symmetric tensor $C_{a b}$ of conformal weight -2 . But if we multiply $g$ by $\alpha^{-2}$ while changing coordinates by $x^{a} \rightarrow \alpha x^{a}$, where $\alpha \in \mathbb{C} \rightarrow\{0\}$, we have

$$
g_{a b} \mapsto \hat{g}_{a b}=\delta_{a b}+\alpha^{2} r_{(a b)(c d)} x^{x} x^{d}+\alpha^{3} s_{(a b)(c d e)} x^{c} x^{d} x^{e}
$$

while $C_{a b} \mapsto \hat{C}_{a b}=\alpha^{4} C_{a b}$. Thus, $\forall \alpha \in \mathbb{C}-\{0\}$,

$$
h\left(\alpha^{2} r, \alpha^{3} s\right)=\alpha^{4} h(r, s) .
$$

Since $h$ is represented by a power series, this then implies that $h$ is homogeneous of degree 2 in $r$ and is independent of $s$. The components of $h$ are therefore homogeneous polynomials of order 2 in the curvature tensor of $g$, and $g \mapsto C$ defines a homomorphism

$$
\odot^{2}\left(\mathbb{C}_{(A B C D)} \oplus \mathbb{C}_{\left(A^{\prime} B^{\prime} C^{\prime} D^{\prime}\right)}\right) \rightarrow \mathbb{C}_{(A B)\left(A^{\prime} B^{\prime}\right)}[-2]
$$

of $\left.(G L,(2, \mathbb{C}) \times G L(2, \mathbb{C})) / \mathbb{C}^{*}\right)$-modules. Since there is no common irreducible component shared by these two representations, such a homomorphism must vanish. Thus we have

Theorem 9.2. The obstruction to extending the canonical first-order Poisson thickening to fifth order is precisely the Bach tensor.

The sixth order obstruction arises in a rather different manner. Indeed, in contrast to the previous case, there is a non-trivial obstruction group for the extension of Poisson fattenings of $Q$ from type $(5,4)$ to type $(6,5)$, since 


$$
H^{2}\left(\mathbb{P}_{1} \times \mathbb{P}_{1}, \mathcal{O}(1,1) \otimes \odot{ }^{6} \hat{N}^{*}\right)=\mathbb{C}_{(A B C)\left(A^{\prime} B^{\prime} C^{\prime}\right)}[-2] \neq 0
$$

Moreover, this obstruction is related to that of extending a type $(5,5)$ Poisson fattening to type $(6,5)$ by the natural homomorphism

$$
H^{2}\left(\mathbb{P}_{1} \times \mathbb{P}_{1}, \mathcal{O}(-5,-5)\right) \rightarrow H^{2}\left(\mathbb{P}_{1} \times \mathbb{P}_{1}, \mathcal{O}(1,1) \otimes \odot^{6} \hat{N}^{*}\right)
$$

induced by the inclusion map $\mathcal{O}(-5,5) \subset \mathcal{O}(1,1) \otimes \odot^{6} \hat{N}^{*}$; since this is the Serredual adjoint of

$$
H^{0}\left(\mathbb{P}_{1} \times \mathbb{P}_{1}, \mathcal{O}(-1,-1) \otimes \odot{ }^{6} \hat{N}\right) \rightarrow H^{0}\left(\mathbb{P}_{1} \times \mathbb{P}_{1}, \mathcal{O}(5,5)\right),
$$

which is fibre-wise non-zero and is an $S L(2, \mathbb{C}) \times S L(2, \mathbb{C})$ equivariant map

$$
\mathbb{C}^{(A B C)\left(A^{\prime} B^{\prime} C^{\prime}\right)}[2] \rightarrow \mathbb{C}^{(A B C)\left(A^{\prime} B^{\prime} C^{\prime}\right)}[2]
$$

it is an isomorphism by Schur's lemma.

Composing this obstruction with the map from 4-jets of metrics to Poisson fattenings of $Q$, we obtain a holomorphic map $\hat{h}$ from 4-jets of metrics

$$
g_{a b}=\delta_{a b}+r_{(a b)(c d)} x^{c} x^{d}+s_{(a b)(c d e)} x^{c} x^{d} x^{e}+t_{(a b)(c d e f)} x^{c} x^{d} x^{e} x^{f}
$$

to trace-free symmetric tensors $D_{(a b c)} \in \bigodot^{2} T^{*}[-2]$. Since the composition of $x^{a} \rightarrow \alpha x^{2}$ with the conformal charge $g \mapsto \alpha^{-2} g$ yields

$$
g_{a b} \mapsto \hat{g}_{a b}=\delta_{a b}+\alpha^{2} r_{(a b)(c d)} x^{c} x^{d}+\alpha^{3} s_{(a b)(c d e)} x^{c} x^{d} x^{e}+\alpha^{4} t_{(a b)(c d e f)} x^{c} x^{d} x^{e} x^{f}
$$

and

$$
D_{(a b c)} \mapsto \hat{D}_{(a b c)}=\alpha^{5} D_{(a b c)},
$$

we have

$$
\hat{h}\left(\alpha^{2} r, \alpha^{3} s, \alpha^{4} t\right)=\alpha^{5} \hat{h}(r, s, t)
$$

for all $\alpha \in \mathbb{C}-\{0\}$. Since $\hat{h}$ is represented by a power series in $(r, s, t)$ it follows that $\hat{h}$ is independent of $t$ and is bilinear in $r$ and $s$.

Now since $\hat{h}$ is invariant under coordinate changes which act trivially to second order, and is also conformally invariant, we may assume, using the conformal normal coordinates of Robin Graham [G], that our coordinates are exponential coordinates and that $R_{a b}=\nabla_{(a} R_{b c)}=0$ at the origin. Then $r$ and $s$ represent, respectively, the Weyl curvature $C_{b c d}^{a}$ and its first covariant derivative $\nabla_{e} C_{b c d}^{a}$. As our obstruction $\hat{h}$ now represents a homomorphism

$$
\left[\mathbb{C}_{(A B C D)} \oplus \mathbb{C}_{\left(A^{\prime} B^{\prime} C^{\prime} D^{\prime}\right)}\right] \otimes\left[\mathbb{C}_{(A B C D) E E^{\prime}} \oplus \mathbb{C}_{\left(A^{\prime} B^{\prime} C^{\prime} D^{\prime}\right) E E^{\prime}}\right] \rightarrow \mathbb{C}_{(A B C)\left(A^{\prime} B^{\prime} C^{\prime}\right)}[-2]
$$

of $S L(2, \mathbb{C}) \times S L(2, \mathbb{C})$-modules, we conclude that

$$
D_{(A B C)\left(A^{\prime} B^{\prime} C^{\prime}\right)}=c_{1} \Psi_{A B C D} \nabla^{D D^{\prime}} \tilde{\Psi}_{A^{\prime} B^{\prime} C^{\prime} D^{\prime}}+c_{2} \tilde{\Psi}_{A^{\prime} B^{\prime} C^{\prime} D^{\prime}} \nabla^{D D^{\prime}} \Psi_{A B C D}
$$

for some universal constants $c_{1}$ and $c_{2}$. But under a conformal change $g \mapsto \Omega^{2} g$ we have

$$
\begin{aligned}
c_{1} & \Psi_{A B C D} \nabla^{D D^{\prime}} \widetilde{\Psi}_{A^{\prime} B^{\prime} C^{\prime} D^{\prime}}+c_{2} \widetilde{\Psi}_{A^{\prime} B^{\prime} C^{\prime} D^{\prime}} \nabla^{D D^{\prime}} \Psi_{A B C D} \\
\mapsto & \Omega^{-2}\left(c_{1} \Psi_{A B C D} \nabla^{D D^{\prime}} \tilde{\Psi}_{A^{\prime} B^{\prime} C^{\prime} D^{\prime}}+c_{2} \tilde{\Psi}_{A^{\prime} B^{\prime} C^{\prime} D^{\prime}} \nabla^{D D^{\prime}} \Psi_{A B C D}\right) \\
& +\Omega^{-2}\left(c_{1}+c_{2}\right) 2 \Upsilon^{D D^{\prime}} \Psi_{A B C D} \tilde{\Psi}_{A^{\prime} B^{\prime} C^{\prime} D^{\prime}},
\end{aligned}
$$

where $r=\Omega^{-1} d \Omega$, so that this is conformally invariant iff $c_{1}=-c_{2}$. This shows 
that $D$ is a universal constant times the Eastwood-dighton tensor

$$
E_{a b c}=\Psi_{A B C D} \nabla^{D D^{\prime}} \widetilde{\Psi}_{A^{\prime} B^{\prime} C^{\prime} D^{\prime}}-\Psi_{A^{\prime} B^{\prime} C^{\prime} D^{\prime}} \nabla^{D D^{\prime}} \Psi_{A B C D}
$$

This universal constant cannot be zero, since the second-order perturbative calculation of Raston and Mason [B-M] shows that the obstruction is non-trivial. Thus we've shown

Theorem 9.3. The obstruction to extending the canonical first order Poisson thickening of an ambitwistor space from fifth order to sixth order is the Eastwood-Dighton tensor.

This concludes the proof of the Main Theorem.

\section{Problems and Perspectives}

We conclude this paper by indicating some of the open problems suggested by this investigation.

(a) Conformal Supergravity. Given a Poisson-thickened ambitwistor space $\mathscr{N}^{(m)}$, we can build an associated complex supermanifold $\mathscr{N}^{[m]}$ of dimension $(5 \mid 2 \mathrm{~m})$ as follows. Let $\mathcal{O}_{(m)}(1,1)$ be the "divisor line bundle" of $\mathscr{N} \subset \mathscr{N}^{(m)}$ constructed by the Poisson structure, and notice that $\mathcal{O}_{(m)}(1,1)$ has a canonical section $\sigma$ vanishing along $\mathscr{N}$. Let $\mathcal{O}_{(m)}(0,1)$ and $\mathcal{O}_{(m)}(1,0)$ be extensions of $L_{ \pm}$to $\mathscr{N}^{(m)}$, and let $\mathfrak{B}$ be a complex vector space of dimension $m$. Then

$$
\Lambda^{\bullet}\left[\mathfrak{B} \otimes \mathcal{O}_{(m)}(-1,0) \oplus \mathfrak{B}^{*} \otimes \mathcal{O}_{(m)}(0,-1)\right] \otimes \mathcal{O}_{(m)}(1,1)
$$

has a canonical section $\hat{\sigma}=\mathrm{id} \otimes \sigma$, where

$$
\operatorname{id} \in \operatorname{Hom}(\mathfrak{B}, \mathfrak{B})=\mathfrak{B}^{*} \otimes \mathfrak{B} \subset \Lambda^{2}\left[\mathfrak{B} \oplus \mathfrak{B}^{*}\right] .
$$

Thus $\hat{\sigma}$ generates an even ideal $\mathscr{J}$ in

$$
\Lambda^{\bullet}\left[\mathfrak{B} \otimes \mathcal{O}_{(m)}(-1,0) \otimes \mathfrak{B}^{*} \otimes \mathcal{O}_{(m)}(0,-1)\right],
$$

i.e. for every local trivialization of $\mathcal{O}_{(m)}(1,1), \hat{\sigma}$ gives a section of this bundle, and changing trivialization just multiplies this section by an element of $\mathcal{O}_{(m)}$. Thus

$$
\mathscr{N}^{[m]}=\left(\mathcal{N},\left(\Lambda^{\bullet}\left[\mathfrak{B} \otimes \mathcal{O}_{(m)}(-1,0) \oplus \mathfrak{B}^{*} \otimes \mathcal{O}_{(m)}(0,-1)\right]\right) / \mathscr{J}\right)
$$

is a well-defined $\mathbb{Z}_{2}$-graded complex ringed space. Moreover, one can check that $\mathscr{N}^{[m]}$ is a complex supermanifold - i.e. its structure sheaf is locally isomorphic to $\mathcal{O}\left(\Lambda^{\cdot} \mathbb{C}^{2 m}\right)$. The nilpotents of $\mathcal{O}_{(m)}$ have "become" the nilpotents of $\Lambda^{\cdot}\left(\mathfrak{B} \otimes \mathfrak{B}^{*}\right)$ !

We can now create a complex supermanifold $\mathscr{M}^{[m]}$ of dimension $(4 \mid 4 m)$, defined as the complete analytic family of 2 -quadrics in $\mathscr{N}^{[m]}$; this means that there is a double fibration

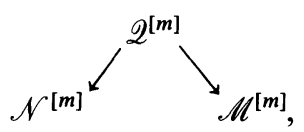

where $\mathscr{2}^{[m]}$ is a supermanifold of dimension $(6 \mid 4 m)$ extending the quadratic bundle $\mathscr{Q}$ and such that any map $\mathbb{P}_{1} \times \mathbb{P}_{1} \times \mathscr{U} \rightarrow \mathscr{N}^{[m]}, \mathscr{U}$ may supermanifold, is induced 
by some map $\mathscr{U} \rightarrow \mathscr{M}^{[m]}$. (Recall that a map of supermanifolds means a map of the underlying "even" manifolds plus a pull-back homomorphism for superfunctions.) The supermanifold $\mathscr{M}^{[m]}$ then has a certain amount of geometric structure - namely the tangent sheaf $T \mathscr{M}^{[m]}$ fits into an exact sequence

$$
0 \rightarrow\left(\mathbf{S}_{+} \otimes E\right) \oplus\left(\mathbf{S}_{-} \otimes E^{*}\right) \rightarrow T \mathscr{M}^{(m)} \rightarrow \mathbf{S}_{+} \otimes \mathbf{S}_{-} \rightarrow 0
$$

of vector bundles (locally free sheaves) on $\mathscr{M}^{[m]}$. This may be called a "superconformal structure," and automatically satisfies certain integrability conditions by virtue of the construction. (Useful background on this story may be found in [M].)

From this point of view, $\mathscr{N}^{[m]}$ becomes a family of certain $(1,2 m)$-dimensional submanifolds of $\mathscr{M}^{[m]}$ which were dubbed super light rays by Witten [Wi1, Wi2]. In linearized theory, the integrability conditions have been studied by Chau and Lim $[\mathrm{C}-\mathrm{L}]$, and seem to correspond precisely to the conformal Einstein system studied here.

It seems plausible that the correct supersymmetric analog of the Poisson structure of $\mathscr{N}^{(m)}$ should be a super contact structure on $\mathscr{N}^{[m]}$, meaning of course of a twisted 1-form which is maximally non-integrable. In the flat case, $\mathscr{N}^{[m]}$ becomes the subvariety of $\mathbb{P}_{3 \mid m} \times \mathbb{P}_{3 \mid m}^{*}$ given by

$$
\sum_{j=1}^{4} Z^{j} W_{j}+\sum_{\mu=1}^{m} \eta^{\mu} \zeta_{\mu}=0
$$

and the super contact form is given by

$$
\theta=W_{j} d Z^{j}+\eta^{\mu} d \zeta_{\mu}
$$

linearized deformations of this structure would then be given by $H^{1}\left(\mathcal{O}_{[m]}(1,1)\right)$, just as linearized Poisson deformations are given by $H^{1}\left(\mathcal{O}_{(m)}(1,1)\right)$. It thus seems certain that the study of the present problem from a supersymmetric viewpoint will lead to new insights and perhaps simplified proofs.

(b) Torsion Theories. We have seen in this paper that the introduction of Poisson structures provides a marvelous tool for fighting through the thicket of obstructions to thickening $\mathscr{N}$. Nonetheless, the equivalence theorem told us that we could, in principle, have done without this crutch - at least if we had been wise enough to choose the right first-order thickening for some other reason! One may well ask, then, if there are similar results to be had concerning torsion geometries; here $\mathscr{N}$ will cease to be a contact manifold, but nonetheless many results would seem to carry over. This point of view has been emphasized by Isenberg and Yasskin [I-Y].

Of course the first problem encountered in such a problem would be to determine whether there is a preferred analog of $\mathscr{N}^{(1)}$ in the presence of torsion. Perhaps one might instead choose to first study the alterations in the obstructions that might occur if we considered a contact ambitwistor space $\mathscr{N}$ with a first-order thickening $\mathscr{N}^{(1)}$ of the non-Poisson type.

In a similar vein, one might investigate the supersymmetric case without taking $\mathcal{N}^{[m]}$ to be super contact. This should allow super geometries with degrees of freedom analogous to classical torsion.

(c) Electromagnetic Coupling. We have chosen in the present work to basically consider only the canonical first order thickening because it made the current 
calculations more tractable. Are the currents different if $\mathscr{L}_{+}$is replaced by another line bundle of the same Chern class? For instance, does the electromagnetic stress-energy tensor couple to the Bach current?

(d) Local Twistors. It seems very likely that it is no accident that the obstructions $B_{a b}$ and $E_{a b c}$ we have encountered are precisely the obstructions that one would expect to encounter in extending the Ward transform of the local twistor connection to $\mathscr{N}^{(4)}$ à la $[\mathrm{I}-\mathrm{Y}-\mathrm{G}]$; cf. [B-M]. Understanding this connection would no doubt link the present work with the Einstein bundle of [L4], which is at present, alas, chiefly notable for its invisibility! Lionel Mason [Ma] has recently announced a program to make this linkage precise.

Acknowledgements. This work is the fruit of many years of useful dialogue with many colleagues. It is a special pleasure to thank Mike Eastwood, whose ideas foreshadowed the jet-bundle construction of Sect. 4 and whose lucid understanding of extension problems and the Penrose transform stimulated much of this research. Then, too, I must offer my warmest thanks to Lionel Mason and Rob Baston, whose ideas exert an almost ubiquitous influence herein. In addition, Alan Weinstein's comments on Poisson structures helped me greatly, as did Roger Penrose's remarks on the rôle of symplectic geometry in twistor theory. Finally, I'd like to thank Dottie Bivona for typing the damn thing.

\section{References}

[B] Bach, R.: Zur Weylschen Relativitätstheorie und der Weylschen Erweiterung des Krummentensorbegriffs. Math. Zeit. 9, 110-135 (1921)

[B-M] Baston, R., Mason, L.: Conformal gravity, the Einstein Equations and spaces of complex null geodesics. Class. Quantum. Grav. 4, 815-826 (1987)

[Bu] Buchdahl, N.: On the relative deRham sequence. Proc. AMS 87, 363-366 (1983)

[C-L] Chau, L.-L. Lim, C.-S.: Geometrical constraints and equations of motion in extended supergravity. Phys. Rev. Lett. 56, 294-297 (1986)

[D] Dighton, K.: An introduction to the theory of local twistors. Int. J. Theor. Phys. 11, $31-43$ (1974)

[E-L] Eastwood, M., LeBrun, C.: Thickenings and supersymmetric extensions of complex manifolds. Am. J. Math. 108, 1177-1192 (1986)

[E-L2] Eastwood, M., LeBrun, C.: Fattening complex manifolds. Preprint (1989)

[G] Graham, R.: Private communication

[I-Y] Isenberg, J., Yasskin, P.: Non-self-dual nonlinear graviatons. Gen. Rel. Grav. 14, 621-627 (1982)

[I-Y-G] Isenberg, J., Yasskin, P., Green, P.: Non-self-dual Gauge Fields. Phys. Lett. 78B, 462-464 (1978)

[K-N-T] Kozameh, C., Newman, E. T., Tod, K. P.: Conformal Einstein spaces. Gen. Rel. Grav. 17, 343-352 (1985)

[L1] LeBrun, C.: The first formal neighborhood of ambitwistor space for curved space-time. Lett. Math. Phys. 6, 345-354 (1982)

[L2] LeBrun, C.: Spaces of complex null geodesics in complex-Riemannian Geometry. Trans. AMS 278, 209-231 (1983)

[L3] LeBrun, 3.: Ambitwistors and Einstein's Equations. Class. Quantum Grav. 2, 555-563 (1985)

[L4] LeBrun, C.: Thickenings and gauge fields. Class. Quantum. Grav. 3, 1039-1059 (1986)

[M] Manin, Y. I.: Gauge Field Theory and Complex Geometry. Berlin, Heidelberg, New York: 1988; translated from the Russian. Kalibrovochnye polya i kompleksnaya geometriya. Moscow: Nauka, 1984 by Koblitz, N., King J

[Ma] Mason, L.: Private communication 
[P] Penrose, R.: Non-linear gravitons and curved twistor theory. Gen. Rel. Grav. 7, 31-52 (1976)

[P-R] Penrose, R. Rindler, W.: Spinors and Space-Time. Vol. 2. Cambridge, London, New York: Cambridge Univ. Press 1986

[W] Weinstein, A.: The local structure of Poisson manifold. J. Diff. Geom. 18, 523-557 (1983)

[Wi1] Witten, E.: An interpretation of classical Yang-Mills theory. Phys. Lett. 77B, 394-398 (1978)

[Wi2] Witten, E.: Twistor-like transform in ten dimensions. Nucl. Phys. B266 245-264 (1986)

Communicated by S.-T. Yau 
